\title{
Predicting a contact's sensitivity to initial conditions using metrics of frictional coupling
}

\author{
R.C. Flicek ${ }^{a 1}$, M.R.W Brake ${ }^{b}$, and D.A. Hills ${ }^{c}$ \\ ${ }^{a}$ Akima Infrastructure Services, under contract to Sandia National Laboratories ${ }^{\dagger}$ \\ PO Box 5800, Albuquerque, NM 87185, USA \\ ${ }^{b}$ Sandia National Laboratories ${ }^{2}$ \\ PO Box 5800, Albuquerque, NM 87175, USA \\ ${ }^{c}$ Department of Engineering Science, University of Oxford, \\ Parks Road, Oxford OX1 3PJ
}

\begin{abstract}
This paper examines two metrics of frictional coupling, which are then used to predict how sensitive a frictional contact's steady-state behavior is to its initial conditions. Based on a large set of numerical simulations with different contact geometries, material combinations, and friction coefficients, a contact's sensitivity to initial conditions is found to be correlated with the product of the coupling metric and the friction coefficient. For cyclic shear loading, this correlation is maintained for simulations with different contact geometries, material combinations, and friction coefficients. However, for cyclic bulk loading, the correlation is only maintained when the contact edge angle is held constant.
\end{abstract}

Keywords: frictional coupling; fretting; shakedown; contact

\section{Introduction}

Engineered structures often comprise multiple components that are fastened together at frictional interfaces, and these interfaces are frequently subjected to a static load superposed with a oscillatory load (e.g. due to vibrations). Applications in which these loading conditions arise include riveted and bolted joints [1,2], dove-tail connections in jet engines [3, 4], and spline couplings [5, 6]. Although the interfaces in components such as these usually appear to be stuck together, zones of micro-slip commonly develop

\footnotetext{
${ }^{1}$ Corresponding author: R.C. Flicek. Email: rcflice@sandia.gov.

${ }^{2}$ Sandia National Laboratories is a multi-mission laboratory managed and operated by Sandia Corporation, a wholly owned subsidiary of Lockheed Martin Corporation, for the U.S. Department of Energy's National Nuclear Security Administration under contract DE-AC04-94AL85000.
} 
at the edges of contact during cyclic loading. This has two main effects: (i) it results in the well-known damage process of fretting fatigue, which significantly reduces the service life of engineering components [7]; and (ii) it provides a source of damping, which can improve system performance [8,9]. Because of these effects, it is important for engineers to be able to predict the slip-stick behavior of frictional interfaces.

Although contact problems involving friction are history dependent, several studies have demonstrated that various frictional systems approach a steady-state response when subjected to periodic loading $[10,11,12,13]$. This occurs due to the process of frictional shakedown: namely, the slip that occurs in the first few cycles generates residual shear tractions that act to reduce the amount of slip in subsequent cycles. Thus, the range of slip-stick behavior that is possible after several load cycles is more restricted than that which may occur during the initial transient phase of loading. Hence, for systems that are likely to spend the majority of their service life in approximately steady-state conditions, e.g. contacts subject to high-cycle fatigue, significant computational savings can be achieved by avoiding analysis of the system's transient behavior and focusing instead on its steady-state response.

A contact's steady-state response of course depends on its geometry and the loading regime, but it can also depend on the initial conditions. This sensitivity to initial conditions is somewhat problematic because it means that, for example, the way a component is installed can influence its steady-state response, changing the amount of damping it produces and fretting damage it incurs. Recent work on frictional shakedown suggests that a contact's sensitivity to initial conditions is related to the level of coupling in the system: that is, the degree to which slip displacements influence the normal pressure distribution. For example, Klarbring et al. [14] and Barber et al. [15] have shown that initial conditions have no influence on whether an uncoupled contact shakes down, i.e. eventually reach a non-dissipative steady-state response in which no slip occurs. In addition, for uncoupled contacts that can adequately be modelled as half-planes, Barber et al. [12] have shown that all of the time varying terms in the steady-state solution (hence the energy dissipated per cycle due to friction) are independent of initial conditions.

In contrast, initial conditions generally influence the steady-state response when coupling is present. Hence, for coupled contacts, there generally exists a range of cyclic load amplitudes for which the initial conditions determine whether the contact shakes down $[12,14,15,16]$, which is referred to as the conditional region. The lower and upper bounds of this region are denoted $\lambda_{1}$ and $\lambda_{2}$, respectively, where $\lambda$ is a dimensionless load factor, i.e. the ratio of the cyclic load amplitude to the static load. Thus, shakedown is guaranteed for $\lambda<\lambda_{1}$, it is impossible for $\lambda>\lambda_{2}$, and it depends on initial conditions for $\lambda_{1}<\lambda<\lambda_{2}$. Note that $\lambda_{1}$ and/or $\lambda_{2}$ may be zero, implying either that shakedown is impossible (when $\lambda_{2}=0$ ) or that it is sometimes possible but is never guaranteed (when $\lambda_{1}=0, \lambda_{2}>0$ ). Also notice that the results presented in $[14,15]$ are equivalent to the statement that $\lambda_{1}=\lambda_{2}$ for uncoupled contacts. 
Determining the loading regimes for which the steady state is certain to be dissipative (i.e. when $\lambda>\lambda_{2}$ ) or non-dissipative (i.e. when $\lambda<\lambda_{1}$ ) is clearly of practical importance. A method for calculating $\lambda_{1}$ and $\lambda_{2}$ is presented by Ahn et al. [16], which involves solving numerous linear systems of equations. While this approach can easily be applied to relatively small systems (e.g. the 10 node system in [13]), it would be computationally prohibitive to apply it to realistic finite element models, which incorporate one or two orders of magnitude more contact nodes. Fortunately, the calculation of $\lambda_{2}$ can be posed as a constrained optimization problem for which many efficient solution algorithms exist [17, 18], enabling $\lambda_{2}$ to be determined for quite large systems. Unfortunately, an efficient method for calculating $\lambda_{1}$ is not yet known. However, because $\lambda_{2}$ can be calculated efficiently, $\lambda_{1}$ could be determined if the size of the conditional region could be found.

Since initial conditions only influence whether shakedown occurs when coupling is present, one might expect the degree to which the steady state depends on initial conditions to be related to the level of coupling in the system. In other words, increasing the level of coupling should increase the size of the conditional region. However, to assess this hypothesis, a metric for quantifying how much coupling is present in a frictional system must first be determined.

Broadly speaking, coupling can either be quantified in a point-wise sense at each location in the system or in an aggregate sense for the entire system. For example, Klarbring et al. [14] presented a type of point-wise coupling metric for discrete systems in which a coupling coefficient is assigned to each node based on the normal reaction force resulting from a tangential displacement distribution. However, when coupling is quantified in a point-wise sense, it can be difficult to compare the level of coupling in different systems with different coupling distributions. For this reason, the two coupling metrics presented by Brake et al. [19] are examined here, which quantify the overall level of coupling in a discrete frictional system as a single non-negative scalar.

The aim of this paper is: (i) to assess the implications of the coupling metrics presented in [19] and (ii) to use these metrics to predict the size of the conditional region. The structure of this paper is as follows. In $\S 2$, the discrete contact problem is formulated, and the coupling metrics are defined. In $\S 3$, these coupling metrics are then calculated for several different contact geometries, and their implications are examined. In $\S 4$, the relationship between coupling and a contact's sensitivity to its initial conditions is examined by performing numerous transient simulations for several example problems. Finally, the implications of these results are discussed in $\S 5$.

\section{Formulation}

Consider a two-dimensional contact that is discretized using the finite element method such that there are $N$ nodes in potential frictional contact along the interface. The reaction forces, $\boldsymbol{r}$, and the relative displacements, $\boldsymbol{u}$, at the contact nodes can be written 
as

$$
\begin{aligned}
\boldsymbol{r} & =[\boldsymbol{q}, \boldsymbol{p}]^{T}=\left[q_{1}, \ldots, q_{N}, p_{1}, \ldots, p_{N}\right]^{T} \\
\boldsymbol{u} & =[\boldsymbol{v}, \boldsymbol{w}]^{T}=\left[v_{1}, \ldots, v_{N}, w_{1}, \ldots, w_{N}\right]^{T},
\end{aligned}
$$

where $q_{i}, p_{i}$ are the shear, normal contact forces, and $v_{i}, w_{i}$ are the tangential, normal relative displacements, where $i \in\{1, \ldots, N\}$. Note that $p_{i}$ is taken to be positive in compression (the negative y-direction), $w_{i}$ is positive for a positive gap (the positive $y$ direction), and $q_{i}, v_{i}$ are positive in the positive $\mathrm{x}$-direction with respect to the Cartesian coordinate systems shown in Figs. 4 and 6.

The contact forces can be written as

$$
\boldsymbol{r}(t)=\boldsymbol{K} \boldsymbol{u}(t)+\boldsymbol{r}^{w}(t),
$$

where $t$ represents time, $\boldsymbol{K}$ is a symmetric $2 N \times 2 N$ contact stiffness matrix, and $\boldsymbol{r}^{w}$ accounts for the influence of the external loads on the contact forces. Specifically, $\boldsymbol{r}^{w}$ represents the forces that would be generated by the external loads if all of the contact displacements were set to zero, i.e. if $\boldsymbol{u}=\mathbf{0}$. Note that both $\boldsymbol{K}$ and $\boldsymbol{r}^{w}$ can be obtained from a standard finite element model using the static reduction procedure described in [20]. Also note that the contact stiffness matrix, $\boldsymbol{K}$, is positive semi-definite (and singular) if either contacting body has rigid body degrees of freedom; otherwise $\boldsymbol{K}$ is positive definite.

By grouping normal and tangential terms, Eq. (2) can be re-written in the form

$$
\left\{\begin{array}{l}
\boldsymbol{q}(t) \\
\boldsymbol{p}(t)
\end{array}\right\}=\left[\begin{array}{cc}
\boldsymbol{A} & \boldsymbol{B}^{T} \\
\boldsymbol{B} & \boldsymbol{C}
\end{array}\right]\left\{\begin{array}{c}
\boldsymbol{v}(t) \\
\boldsymbol{w}(t)
\end{array}\right\}+F(t)\left\{\begin{array}{l}
\boldsymbol{q}^{w} \\
\boldsymbol{p}^{w}
\end{array}\right\},
$$

where $\boldsymbol{q}, \boldsymbol{p}, \boldsymbol{v}, \boldsymbol{w}$ are defined in Eq. (1), $F(t)$ is a function that describes how the applied loads vary with time, and $\boldsymbol{K}$ is partitioned into sub-matrices corresponding to the following force-displacement relationships: tangential-tangential, $\boldsymbol{A}$; normal-tangential, $\boldsymbol{B}$; and normal-normal, $\boldsymbol{C}$. Note that since $\boldsymbol{K}$ is symmetric and positive semi-definite, so are $\boldsymbol{A}$ and $\boldsymbol{C}$, whereas $\boldsymbol{B}$ generally is not symmetric.

\subsection{Coupling metrics}

Equation (3) illustrates that $\boldsymbol{B}$ accounts for the effects of coupling in the system: namely, it quantifies the normal contact forces that are generated by tangential slip displacements. In contrast, $\boldsymbol{A}$ determines the tangential contact forces resulting from tangential displacements. Thus, a measure of the level of coupling can be obtained by taking the ratio of some property of the $\boldsymbol{A}$ and $\boldsymbol{B}$ matrices. In physical terms, this provides a measure of the system's stiffness due to coupling (i.e. normal forces due to tangential displacements) relative to its tangential stiffness (i.e. shear forces due to tangential displacements). 
Two coupling metrics are proposed in [19], which are based on: (i) the L2-norm, $\xi_{\text {norm }}$, and (ii) the eigenvalue with the largest modulus, $\xi_{\text {eig }}$, which can be written as

$$
\begin{aligned}
\xi_{\text {norm }} & =\frac{\|\boldsymbol{B}\|_{2}}{\|\boldsymbol{A}\|_{2}} \\
\xi_{\text {eig }} & =\frac{|\operatorname{eig}(\boldsymbol{B})|_{\max }}{|\operatorname{eig}(\boldsymbol{A})|_{\max }} .
\end{aligned}
$$

These coupling metrics may be collectively referred to as $\xi$ or individually with the appropriate subscript. Note that since $\boldsymbol{B}$ generally is not symmetric, its eigenvalues are usually complex; hence the numerator of Eq. (4b) generally involves calculating the modulus of a complex number. Also note that both coupling metrics are non-negative because both the L2-norm of a matrix and the modulus of a real/complex number are non-negative. Of course, numerous other metrics of this form could also be used, e.g. based on different matrix norms.

\subsubsection{Properties of the coupling metrics}

Notice that both coupling metrics in Eq. (4) are based on properties of the submatrices of $\boldsymbol{K}$. Furthermore, as noted in [20], $\boldsymbol{K}$ is the solution to the contact problem when the prescribed loading conditions are replaced by the equivalent homogeneous conditions. Hence, when the loads are prescribed in force or displacement control, zeroforce or zero-displacement conditions, respectively, are introduced to the problem. For this reason, $\boldsymbol{K}$ is unaffected by loads that are applied in force control, but $\boldsymbol{K}$ is modified if displacement-controlled loads are applied (since these introduce zero-displacement boundary conditions, which affect the system's stiffness). Consequently, if the loads are applied in force control, both coupling metrics defined in Eq. (4) are a property of the system's geometry alone: that is, they are identical for all force-controlled loading regimes.

\section{Assessment of the coupling metrics}

In frictional contact problems, coupling arises from two sources: (i) material mismatch and (ii) domain mismatch. Material mismatch occurs when the material properties of the two elastic bodies are not identical, whereas domain mismatch results from the two bodies being of a different shape. In order to investigate the implications of the coupling metrics systematically, elastic mismatch and domain mismatch are examined separately.

\subsection{Material mismatch}

For the most general contact problem between two linear-elastic, isotropic bodies, the stresses within the bodies depend on four material properties, e.g. $\mu_{1}, \mu_{2}, \nu_{1}, \nu_{2}$, 
where $\mu$ is the shear modulus, $\nu$ is Poisson's ratio, and the subscript denotes the body to which the property applies. However, if all of the prescribed loads are applied as surface tractions (or in force control), Dundurs [21] showed that the stress state only depends on two composite parameters, which are defined as [22]

$$
\alpha=\frac{\frac{\kappa_{1}+1}{\mu_{1}}-\frac{\kappa_{2}+1}{\mu_{2}}}{\frac{\kappa_{1}+1}{\mu_{1}}+\frac{\kappa_{2}+1}{\mu_{2}}}, \quad \beta=\frac{\frac{\kappa_{1}-1}{\mu_{1}}-\frac{\kappa_{2}-1}{\mu_{2}}}{\frac{\kappa_{1}+1}{\mu_{1}}+\frac{\kappa_{2}+1}{\mu_{2}}},
$$

where $\kappa$ is Kolosov's constant, which is given by

$$
\kappa= \begin{cases}3-4 \nu & \text { for plane strain } \\ \frac{3-\nu}{1+\nu} & \text { for plane stress }\end{cases}
$$

Note that $-1 \leqslant \alpha \leqslant 1$, and $-0.5 \leqslant \beta \leqslant 0.5$ (assuming $0 \leqslant \nu \leqslant 0.5$ ). Also note that $\alpha, \beta=0$ when the contacting bodies have identical material properties. ${ }^{3}$ Finally, a further reduction in the dependence on elastic properties occurs when both contacting bodies can be represented as half-planes in which case the stresses depend solely on $\beta .{ }^{4}$

Recall that the coupling metrics, $\xi$, are defined based on properties of the contact stiffness matrix, $\boldsymbol{K}$, which specifies the contact forces resulting from contact displacements. Furthermore, although the stresses within the bodies (and hence the contact forces along the interface) depend only on $\alpha$ and $\beta$ when the loads are applied in force control, the displacements still depend on all four material properties, e.g. $\mu_{1}, \mu_{2}, \nu_{1}, \nu_{2}$. Thus, it is not clear whether $\boldsymbol{K}$ and $\xi$ exhibit the same reduced dependence on material properties as the stresses.

\subsubsection{Half-plane contacts}

To examine the influence of material dissimilarity on $\xi$, consider contact between the two elastic half-planes shown in Fig. 1 under plane-strain conditions, where both halfplanes have a curved surface profile with a radius of $100 a$, and $a$ is the half-width of the contact area. To calculate $\xi$ for this continuum contact problem, the contact must first be discretized, and the contact stiffness matrix, $\boldsymbol{K}$, must be obtained. For this and all subsequent contacts that are studied in this paper, the discretization was performed using the commercial finite element software ABAQUS/CAE v6.11-1, and $\boldsymbol{K}$ was calculated in MATLAB R2013a using the procedure described in [20]. Note that all of the models that are used in $\S 3$ incorporate 128 uniformly spaced nodes along the contact interface

\footnotetext{
${ }^{3}$ See Johnson [23] $§ 5.1$ for a discussion of Dundurs' parameters.

${ }^{4}$ See Barber [22] $\S 12.7$ or Hills et al. [24] $\$ 2.2$ for more details.
} 


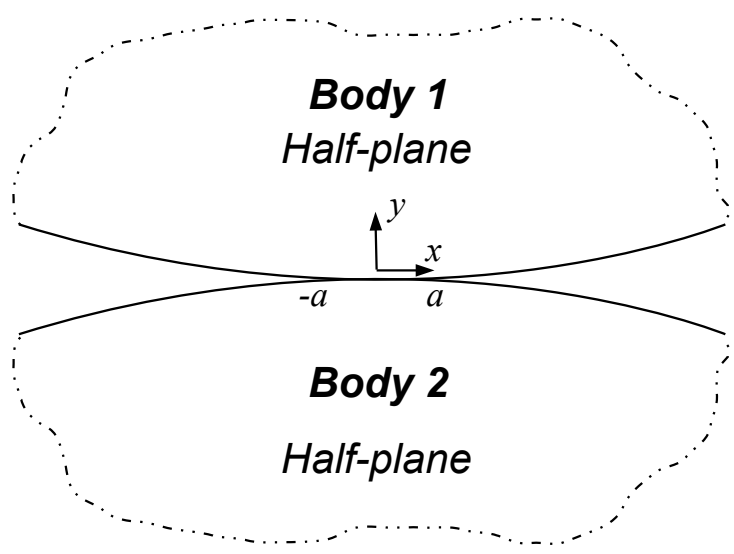

Figure 1: Contact between two elastic half-planes.

based on the results of a convergence study of $\xi .^{5}$

As previously mentioned, material properties only influence the stress state in halfplane contact problems through the composite parameter $\beta$. To investigate whether the coupling metrics, $\xi_{\text {eig }}, \xi_{\text {norm }}$, also display this reduced dependence on material properties, both coupling metrics were computed for a range of material combinations by varying $\alpha$ and $\beta$. The material combinations lie along four lines in $\beta$ vs. $\alpha$ space, which are shown in Fig. 2: namely, $\alpha=1, \alpha=2 \beta, \alpha=\beta$, and $\alpha=0$. The values of $\alpha$ and $\beta$ resulting from contact between some common materials (which are listed in Table 1) are also shown in Fig. 2.

Fig. 2 also shows the range of admissible material combinations, which is bounded by the parallelogram about the origin. Note that $\alpha=1$ when body 2 is rigid; $\alpha=2 \beta$ when $\nu_{1}, \nu_{2}=0 ; \alpha=\beta$ when $\mu_{2} / \mu_{1}=1$ (when the bodies have the same elastic modulus); and $\beta=0$ when $\nu_{1}, \nu_{2}=0.5$ (when both bodies are incompressible). Also note that swapping the labeling of the two bodies simply inverts the signs of $\alpha$ and $\beta$.

Figure 3 shows $\xi_{\text {eig }}$ and $\xi_{\text {norm }}$ plotted against $\beta$ for a range of material combinations. Note that results are only shown for non-negative values of $\beta$ because the results are the same for $\pm \beta$ (since both bodies are geometrically identical). The most striking feature of this figure is that both coupling metrics are approximately equal to $|\beta|$. In addition, both coupling metrics are insensitive to $\alpha$ and other material properties (i.e. $\nu_{1}$ and $\nu_{2}$ ). ${ }^{6}$ Thus, Fig. 3 illustrates that both metrics describe the level of coupling due to material

\footnotetext{
${ }^{5}$ See $\S$ Appendix A.3.1 for a convergence study of the steady-state slip displacement.

${ }^{6}$ The authors attribute the minor dependence of the coupling metrics on these other material properties to numerical inaccuracies associated with the discretization.
} 


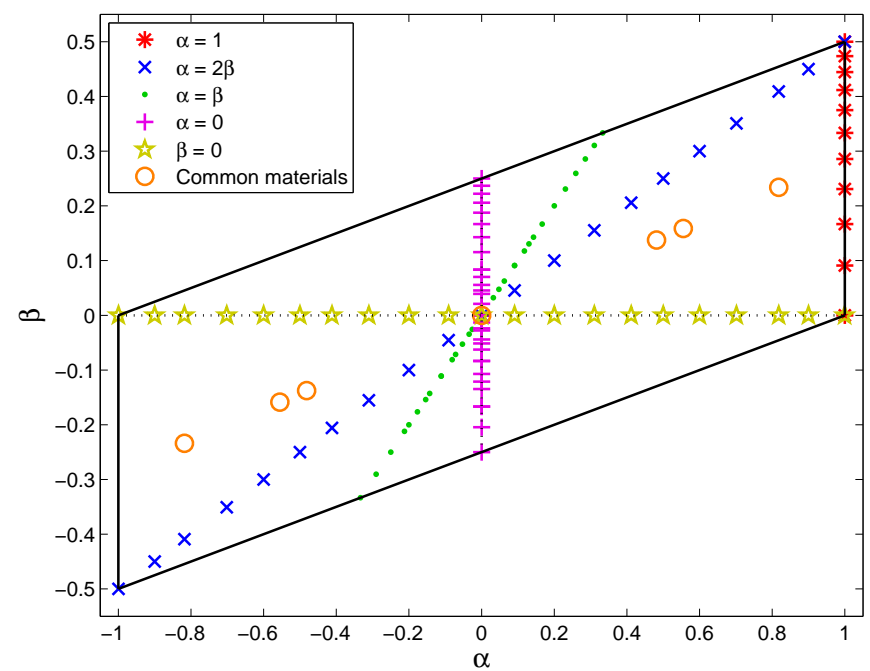

Figure 2: A plot of admissible material combinations in $\beta$ vs. $\alpha$ space.

\begin{tabular}{|cccccccc|}
\hline Name & Material $_{1}-$ Material $_{2}$ & $\alpha$ & $\beta$ & $E_{1}$ & $E_{2}$ & $\nu_{1}$ & $\nu_{2}$ \\
\hline StSt & Steel-Steel & 0 & 0 & 200 & 200 & 0.3 & 0.3 \\
AlSt & Aluminum-Steel & 0.4815 & 0.1376 & 70 & 200 & 0.3 & 0.3 \\
StTc & Steel-Tungsten Carbide & 0.5556 & 0.1587 & 200 & 700 & 0.3 & 0.3 \\
AlTc & Aluminum-Tungsten Carbide & 0.8182 & 0.2338 & 70 & 700 & 0.3 & 0.3 \\
\hline
\end{tabular}

Table 1: Material properties used for the results shown in Fig. 12. Note that the values of $E_{1}, E_{2}$ are given in $G P a$.

mismatch in half-plane problems accurately and that coupling is strongly influenced by $\beta$.

\subsubsection{Common-edged contacts}

Given that the coupling metrics are mainly determined by $\beta$ for half-plane contacts, the influence of $\alpha$ on the coupling metrics must be examined using a different contact geometry. In order to exclude the effects of geometric dissimilarity, common-edged contacts are used for this investigation, which consist of contact between two geometrically identical contacts along a plane of symmetry. Three such contacts between parallelograms with edge angles, $\phi$, of $60^{\circ}, 90^{\circ}$, and $120^{\circ}$ are shown in Fig. 4, where $a$ is the half-width of the contact. Note that this figure also shows the applied loads and boundary conditions for the $90^{\circ}$ and $120^{\circ}$ contact angles, which are used in the analysis in $\S 4$.

Both coupling metrics were calculated for these three plane-strain contact geometries for a range of material combinations. Perhaps surprisingly, the values of $\xi$ for material combinations lying along the lines $\alpha=2 \beta, \alpha=\beta$, and $\alpha=0$ are almost identical 


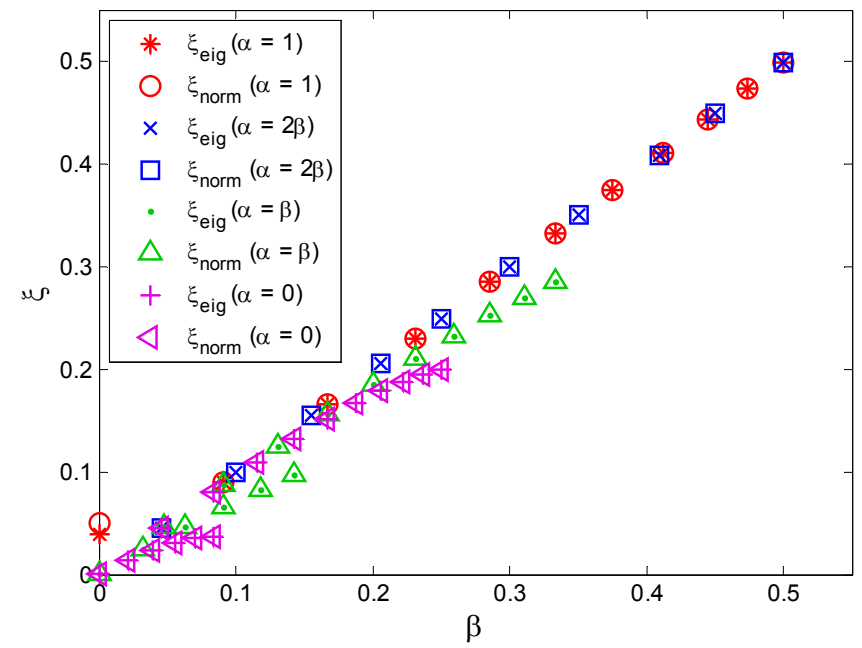

Figure 3: The influence of $\beta$ on the coupling metrics, $\xi_{\text {eig }}, \xi_{n o r m}$, for contact between two elastic halfplanes.

to those for half-plane problems. Of the material combinations shown in Fig. 2, major differences only appear for those that lie along $\alpha=1$ or $\beta=0$. Results for the $\beta=0$ case (when both bodies are incompressible) are shown in Fig. 5, which displays $\xi$ plotted against $\alpha$ for the three common-edged contacts shown in Fig. 4. Note that results are only shown for non-negative $\alpha$ since both bodies are geometrically identical.

Figure 5 illustrates that common-edged contacts are moderately influenced by $\alpha$. However, $\alpha$ has a much smaller effect on $\xi$ than $\beta$ does in all cases. In addition, $\xi_{\text {eig }} \leqslant \xi_{\text {norm }}$, and there is a larger discrepancy between $\xi_{\text {eig }}$ and $\xi_{\text {norm }}$ in Fig. 5 than in Fig. 3. However, it is not clear which metric provides a better assessment of the level of coupling based on this figure. Fig. 5 also suggests that $\alpha$ has a stronger influence on the level of coupling as the edge angle decreases, such that $\xi$ is approximately twice as large for $\phi=60^{\circ}$ as it is for $\phi=120^{\circ}$ when $\alpha=1$.

Note that results for the half-plane problem analyzed in $\$ 3.1 .1$ are also included in Fig. 5 for comparison. Recall that the authors attribute the half-plane's dependence on material properties other than $\beta$ to numerical inaccuracies in the calculations. The most obvious source of error is that the half-planes are modeled as large but finite blocks and not as true half-planes. However, some numerical error is also associated with the calculation of $K$ itself since its calculation requires numerous floating point operations (i.e. matrix multiplications and inversions) to be performed on matrices whose coefficients span many orders of magnitude. Moreover, this error should increase with increasing material dissimilarity (because this will increase the discrepancy in the size of the coefficients of the stiffness matrices).

In Fig. 5, the level of coupling increases gradually with increasing $\alpha$ for the half-plane contact, which provides some evidence that this second source of error 


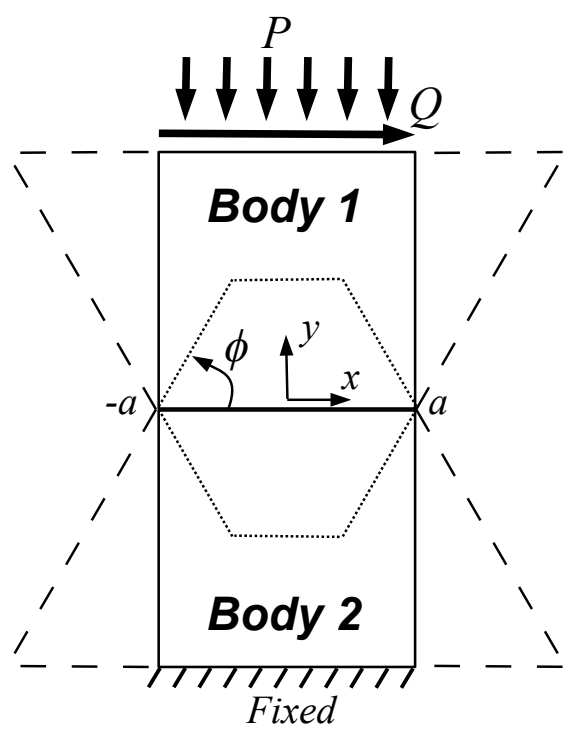

Figure 4: A diagram of three common-edged contacts between parallelograms with edge angles, $\phi$, of $60^{\circ}$ (dotted), $90^{\circ}$ (solid), and $120^{\circ}$ (dashed).

becomes a bit larger than might be expected for problems with high material dissimilarity. Thus, the implications of the results in Fig. 5 taken together are: i) $\alpha$ does have a moderate influence on coupling for non-half-plane contacts, ii) $\alpha$ has a much weaker influence on coupling than $\beta$, iii) numerical error in the calculations increases with increasing material dissimilarity, and iv) for contacts between highly dissimilar materials, the influence of $\alpha$ on $\xi$ is only a few times greater than the influence of numerical error.

\subsection{Domain mismatch}

In order to examine the influence of domain mismatch on the coupling metrics, the three complete contacts shown in Fig. 6 are considered, which are formed between a parallelogram and an elastically similar half-plane, resulting in a contact half-width of $a$. Note that this figure also shows the applied loads for the $90^{\circ}$ and $120^{\circ}$ contact angles, which are used in the analysis in $\S 4$. The coupling metrics for these contacts were calculated for a range of values of $\nu_{1}, \nu_{2}$ (but which all resulted in $\alpha, \beta=0$ ), and $\xi$ was found to be the same in all cases. The results are shown in Table 2, which displays $\xi_{\text {eig }}$ and $\xi_{\text {norm }}$ for the three complete contacts and also for contact between elastically similar half-planes for comparison.

Thus, the coupling metrics suggest, at least for the examples considered here, that geometric dissimilarity results in weaker coupling than does strong material dissimilarity. This is especially so for large values of $\beta$, which results in $\xi$ of up to 0.5 . In comparison, the maximum values of $\xi$ for the domain mismatch cases considered here 


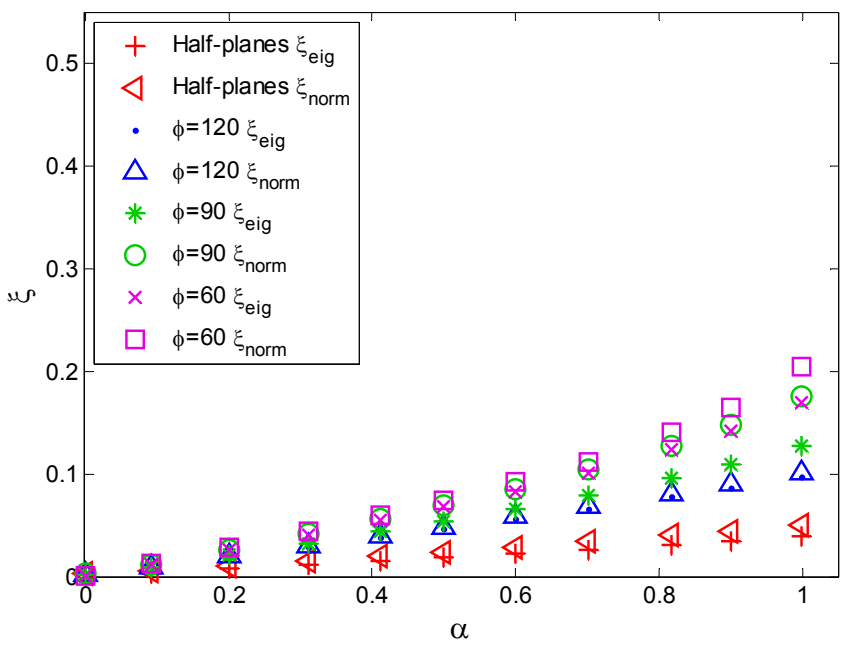

Figure 5: The influence of material mismatch on the coupling metrics, $\xi_{\text {eig }}, \xi_{\text {norm }}$, for common-edged contacts when $\beta=0$.

\begin{tabular}{|l|cccc|}
\hline Metric & $\phi=60^{\circ}$ & $\phi=90^{\circ}$ & $\phi=120^{\circ}$ & Half-planes \\
\hline$\xi_{\text {eig }}$ & 0.140 & 0.098 & 0.061 & 0.001 \\
$\xi_{\text {norm }}$ & 0.185 & 0.145 & 0.064 & 0.003 \\
\hline
\end{tabular}

Table 2: The values of both coupling metrics, $\xi_{\text {eig }}, \xi_{\text {norm }}$, for three complete contacts and one half-plane contact for the elastically similar case when $\alpha, \beta=0$.

are $\xi_{\text {eig }}=0.140$ and $\xi_{\text {norm }}=0.185$ when $\phi=60^{\circ}$. However, Table 2 shows that $\xi$ increases as $\phi$ decreases, which suggests that if smaller edge angles were considered, higher values of $\xi$ would result. Finally, Table 2 shows that there is a larger discrepancy between $\xi_{\text {eig }}$ and $\xi_{\text {norm }}$ for domain mismatch than for material mismatch. Also, as with the previous results, $\xi_{\text {eig }} \leqslant \xi_{\text {norm }}$.

It is perhaps surprising that these coupling metrics suggest that material mismatch results in more coupling than does geometric dissimilarity. However, recall that $\xi$ is a measure of coupling in an aggregate sense. Therefore, it may be the case that domain mismatch results in more severe localized coupling, e.g. at the contact edges, but much less coupling in other regions, e.g. at the interior of the contact. Moreover, if a particular problem were studied in which slip occurred in a localized region of high coupling, this may have a greater impact on the solution than a moderate level of coupling that is spread more uniformly along the interface. Indeed, coupling only influences the problem if slip occurs in the region of the interface where it is present. Hence, this is a potential drawback of using an aggregate measure of coupling as opposed to a point-wise metric. 


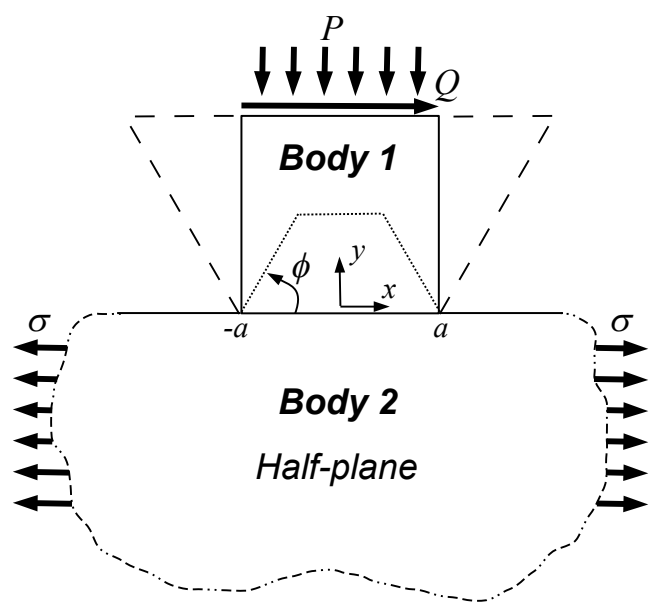

Figure 6: A diagram of three complete contacts formed between a parallelogram with an edge angle, $\phi$, of $60^{\circ}$ (dotted), $90^{\circ}$ (solid), and $120^{\circ}$ (dashed) and a half-plane.

\subsection{Mixed coupling}

In this section, the case when both material mismatch and domain mismatch are present is considered. To do this, material dissimilarity can simply be added to the complete contacts shown in Fig. 6. Results for this mixed-coupling case are plotted in Fig. 7 for $\phi=60^{\circ}, 90^{\circ}$, and $120^{\circ}$. In this figure, the coupling metrics are plotted against $\beta$ for material combinations lying along the lines $\alpha=1, \alpha=2 \beta, \alpha=\beta$, and $\alpha=0$, which are shown in Fig. 2.

Figure 7 illustrates that the effects of domain mismatch are most influential when $\beta$ is small, but as $|\beta|$ increases, $\xi$ becomes dominated by $\beta$. Indeed, once $\beta \gtrsim 0.3$, the influence of domain mismatch is negligible (since the results become nearly identical to those computed for half-plane contacts). However, notice from Fig. 1 and Table 1 that common engineering material combinations rarely result in $\beta>0.25$ (e.g. aluminum in contact with tungsten carbide gives $\beta=0.2338$ ). Thus, within the range of $\beta$ that is likely to be found in practice, domain coupling actually dominates the level of coupling that is present. Figure 7 also illustrates that coupling due to domain mismatch increases with decreasing edge angle, which is in agreement with Table 2. Furthermore, as in Fig. 3, the coupling metrics show only a weak dependence on $\alpha, \nu_{1}, \nu_{2}$ for material combinations along the lines $\alpha=1, \alpha=2 \beta, \alpha=\beta$, and $\alpha=0$.

Figure 8 displays the interaction between domain mismatch and $\alpha$ when $\beta=0$. This figure shows that domain mismatch generally has much more of an influence on $\xi$ than does $\alpha$. The exception to this is when $\alpha \approx-1$, which corresponds to body 1 (the punch) being much stiffer than body 2 (the half-plane). In this situation, the solution approaches the case of a rigid punch contacting an elastic half-plane, so the domain of the punch does not enter the problem. Thus, all three complete contacts (and the half-plane contact as well) result in the same low level of coupling when $\alpha=-1$. 


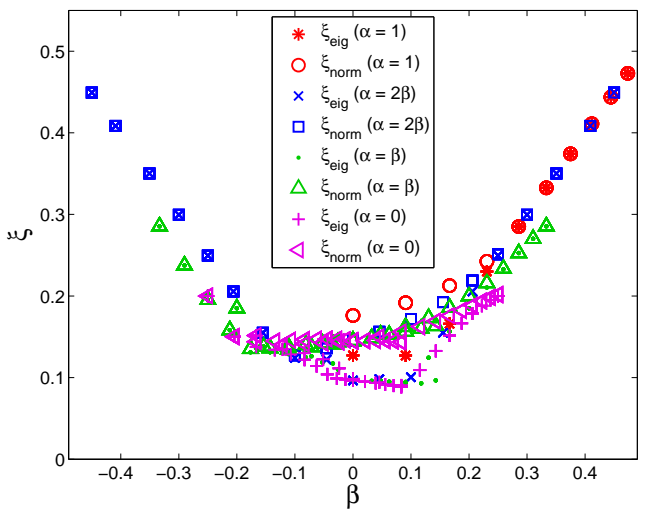

(b)

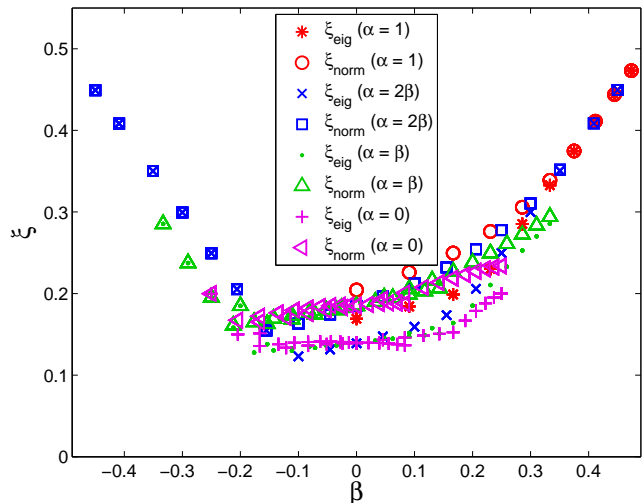

(a)

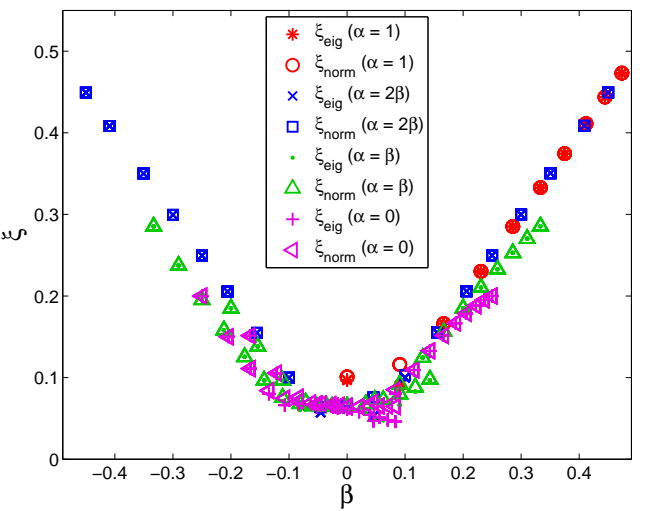

(c)

Figure 7: The influence of domain and material mismatch on the coupling metrics, $\xi_{\text {eig }}, \xi_{\text {norm }}$, for complete contacts with edge angle, $\phi$, of (a) $60^{\circ}$, (b) $90^{\circ}$, and (c) $120^{\circ}$.

Finally, notice that there is a larger discrepancy between $\xi_{\text {eig }}$ and $\xi_{\text {norm }}$ in both Figs. 7 and 8 than in Figs. 3 and 5, which do not incorporate domain mismatch. This discrepancy is largest for contacts with edge angles of $60^{\circ}$ and $90^{\circ}$. Moreover, when the two metrics give different results, $\xi_{\text {eig }}$ consistently gives lower values than $\xi_{\text {norm }}$. Finally, in some cases, $\xi_{\text {norm }}$ varies more smoothly than $\xi_{\text {eig }}$, e.g. when $\phi=90^{\circ}$ in Fig. 8 and $\alpha \approx-0.3$. However, both coupling metrics give similar results in most situations, and their implications are plausible. Thus, both metrics appear to be suitable for quantifying the aggregate level of coupling in frictional systems, though $\xi_{\text {norm }}$ might provide a slightly better assessment due to its smoother variation. 


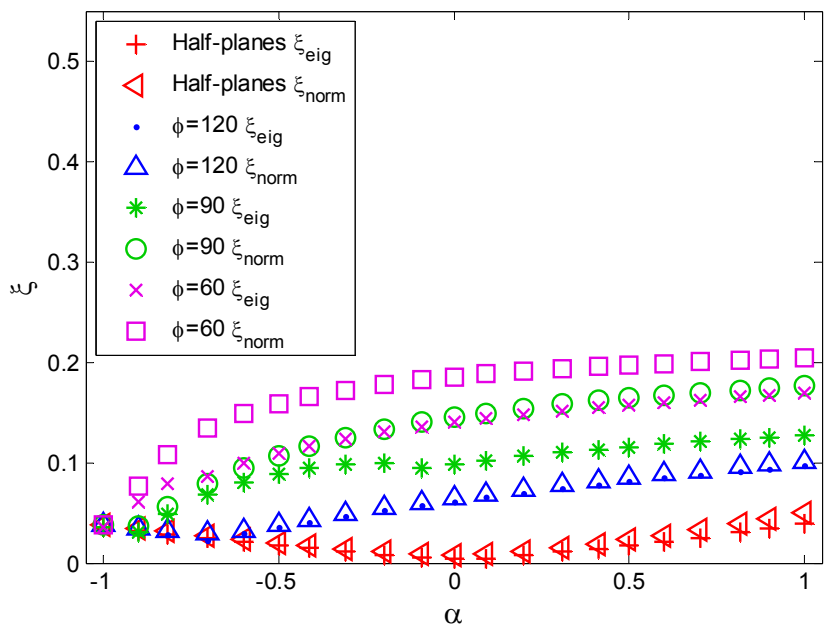

Figure 8: The influence of domain mismatch and $\alpha$ (when $\beta=0$ ) on the coupling metrics, $\xi_{\text {eig }}, \xi_{\text {norm }}$, for complete contacts with edge angle, $\phi$, of $60^{\circ}, 90^{\circ}$, and $120^{\circ}$ and for contact between half-planes.

\section{Sensitivity to initial conditions}

The motivation for developing the coupling metrics presented in $\S 3$ is to provide a computationally inexpensive way of predicting how sensitive a contact's steadystate response is to its initial conditions. Since a contact's steady-state response only depends on initial conditions when coupling is present, it is hypothesized that a contact's initial-condition sensitivity is a function of the amount of coupling in the system. Section 3 shows that the coupling metrics adopted in this paper are most sensitive to material mismatch due to $\beta$, are moderately influenced by domain mismatch, and are insensitive to material mismatch due to $\alpha$. In this section, the influence of these three sources of coupling is examined in the context of several example problems.

The first step in this analysis is to choose a method for quantifying a contact's sensitivity to initial conditions. This can be done in numerous ways, but here the parameter $\Gamma$ is used, which is defined as

$$
\Gamma=1-\frac{\lambda_{1}}{\lambda_{2}}
$$

This parameter quantifies a contact's sensitivity to initial conditions based on the difference between $\lambda_{1}$ and $\lambda_{2}$. Since $\lambda_{1}=\lambda_{2}$ for uncoupled contacts and $\lambda_{2}>\lambda_{1}$ for coupled contacts, $\Gamma$ is zero for uncoupled problems and is positive when coupling is present. One difficulty with using $\Gamma$ as a metric is that it requires both $\lambda_{1}$ and $\lambda_{2}$ to be calculated. Though $\lambda_{2}$ can be calculated easily even for large systems $[17,18]$, no computationally efficient approach currently exists for calculating $\lambda_{1}$. 


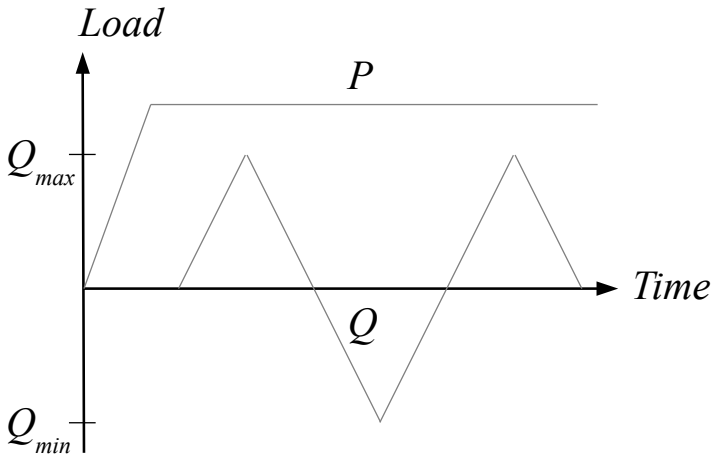

(a)

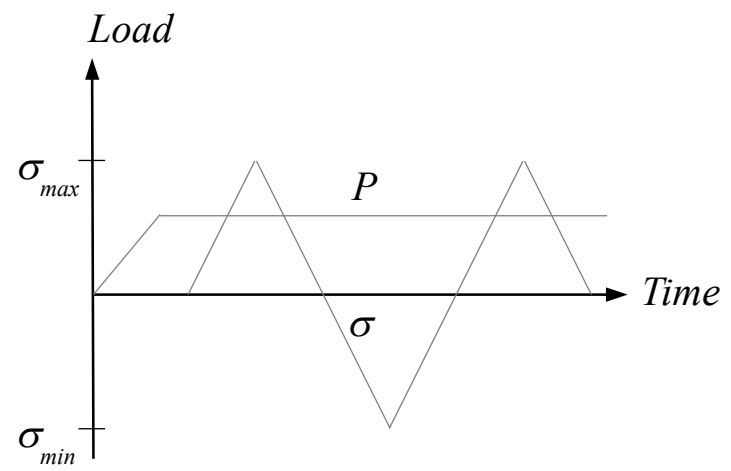

(b)

Figure 9: Diagrams of (a) the cyclic shear loading regime and (b) the cyclic bulk loading regime.

In order to be able to calculate $\Gamma$, the authors developed an algorithm for obtaining an upper-bound estimate of $\lambda_{1}$. Broadly speaking, this algorithm functions by performing a series of marching-in-time simulations, which are run until a steady state is reached. Numerous simulations are performed with different load factors, $\lambda$, and initial displacement conditions, $v_{0}$, while searching for the $v_{0}$ that results in shakedown failing to occur for the lowest $\lambda$. The lowest $\lambda$ that does not result in shakedown provides an upper-bound estimate of $\lambda_{1}$. See Appendix A for a detailed explanation this algorithm.

\subsection{Uncoupled contacts under cyclic shear loading}

In the following sections, $\Gamma$ is determined for several contact problems to study their sensitivity to initial conditions. This is done by calculating $\lambda_{2}$ using the algorithm in [17] and estimating $\lambda_{1}$ with the algorithm described in $\S$ Appendix A. However, prior to applying this approach to coupled problems whose sensitivity to initial conditions is not known, this approach is first applied to an uncoupled contact to ensure that the $\lambda_{1}$ estimation algorithm behaves as expected; that is, that $\lambda_{1}=\lambda_{2}$ when $\xi=0$.

Common-edged contacts are used for this verification because they are unique in that they can be uncoupled and can also shake down. This is because complete contacts are always coupled due to domain mismatch, and half-plane contacts cannot shake down aside from a few special cases. ${ }^{7}$ Thus, for this verification, $90^{\circ}$ and $120^{\circ}$ common-edged contacts ${ }^{8}$ were subjected to the loading regime shown in Fig. 9(a): namely, a constant normal load, $P$, was applied, and then a shear load, $Q$,

\footnotetext{
${ }^{7}$ The special case when a cyclically loaded incomplete contact can shake down is when the loads are applied proportionally, and the unloading curve is the exact reversal of the loading curve [15].

${ }^{8}$ Note that the $60^{\circ}$ edge angle is omitted from all subsequent analysis because the top of the $60^{\circ}$ punch
} 


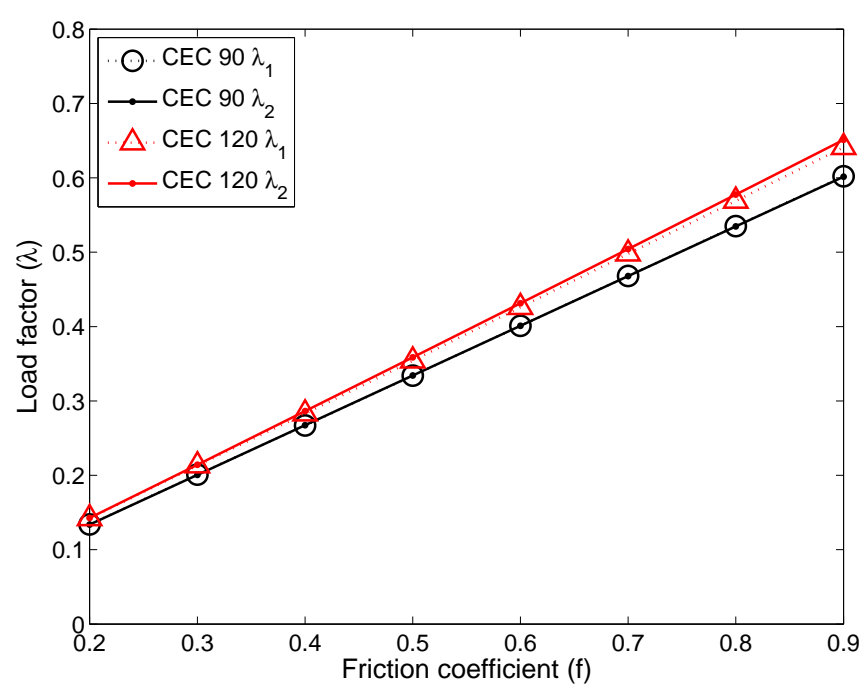

Figure 10: $\lambda_{1}$ and $\lambda_{2}$ plotted against friction coefficient, $f$, for uncoupled common-edged contacts with $\alpha=\beta=0$.

was cycled within the range $\left[Q_{\min }, Q_{\max }\right]$, where $Q_{\min }=-Q_{\max }$. The shear load was cycled until a steady-state response was reached according to the conditions specified in $\S$ Appendix A.1. As shown in Fig. 4, both $P$ and $Q$ were applied over a length of $2 a$ directly above the contact patch, and a zero-displacement condition was applied to the bottom of body 2 directly below the contact patch. Note that $Q$ was applied so as to be statically equivalent to a shear applied through the plane of the interface. This was achieved by applying a linearly varying normal load to the top of the punch to counteract the moment resulting from applying $Q$ at the top of the punch.

The material properties were set to the Steel-Steel case shown in Table 1 so that $\alpha=\beta=0$, making the contact uncoupled. Note that all the results presented in $\S 4$ of this paper were obtained using a model with 64 uniformly spaced contact nodes. This number of nodes was chosen based on the results of the convergence study presented in $\S$ Appendix A.3.1, which shows that this is the minimum number of contact nodes that still provides an adequate description of the interface. ${ }^{9}$ Note that all of the calculations were performed on a PC with a $2.3 \mathrm{GHz}$ processor and $8 \mathrm{~GB}$ of RAM.

The calculation described in [17] was used to calculate $\lambda_{2}$, and the algorithm de-

is smaller than the contact patch, so the loads cannot be applied directly above the contact patch. This makes it difficult to compare the results of the $60^{\circ}$ case to those of the other two angles because the same loading regime cannot be applied.

${ }^{9}$ Note that even with 64 contact nodes, it still takes several hours to estimate $\lambda_{1}$ per friction coefficient, though this is quite problem dependent. For comparison, the calculation of the entire curve of $\lambda_{2}$ vs. $f$ in the range $0.2<f<0.9$ generally takes approximately 1 minute. 
scribed in $\S$ Appendix A was used to obtain an upper-bound estimate of $\lambda_{1}$. A null displacement distribution was used to initialize the algorithm for estimating $\lambda_{1}$, and only one update of the displacement shape was performed using a linear displacement distribution, such that $v_{a}=0$ and $v_{b}=x / a .{ }^{10}$ A linear displacement shape was chosen for $v_{b}$ because it resembles some of the optimal displacement distributions for promoting shakedown that were obtained from the calculation of $\lambda_{2}$ [17]. Note that for the loading regime shown in Fig. 9(a), the load factor, $\lambda$, is defined as the ratio $Q_{\max } / P$, where $Q_{\max }$ is the amplitude (i.e. half the total range) of the cyclic shear load.

The results of these calculations are plotted in Fig. 10, which shows $\lambda_{2}$ and $\lambda_{1}$ vs. the friction coefficient, $f$. In the legends of this and subsequent figures, $\mathrm{CC}$ refers to complete contact, CEC refers to common-edged contact, and 90,120 refer to the $90^{\circ}, 120^{\circ}$ edge angles for either type of contact geometry. Thus, Fig. 10 illustrates that the algorithm does indeed predict the correct response: namely, $\lambda_{1}=\lambda_{2}$ when $\xi=0$. The very minor discrepancies between $\lambda_{1}$ and $\lambda_{2}$ in this figure can be attributed to numerical inaccuracies in the calculations.

\subsection{Coupled contacts under cyclic shear loading}

This section examines the sensitivity to initial conditions of several coupled contacts subjected to the cyclic shear loading shown in Fig. 9(a). Recall that a contact's sensitivity to initial conditions depends on the type of loading regime to which it is subjected, but the coupling metrics only depend on the contact's geometry. Thus, useful comparisons can only be made between contacts that are subjected to similar loading regimes. Note that only the $90^{\circ}$ and $120^{\circ}$ edge angles are considered for the reasons mentioned in $\S 4.1$.

Figure 11 shows $\lambda_{1}$ and $\lambda_{2}$ plotted against $f$ for several coupled contact problems. Figure 11(a) plots results for $90^{\circ}$ and $120^{\circ}$ common-edged contacts between aluminum and steel with the material properties shown in Table 1, such that $\alpha=$ $0.4815, \beta=0.1376$. For both the $90^{\circ}$ and $120^{\circ}$ common-edged contacts, $\xi_{\text {norm }}=$ 0.1373 . Figure 11(b) shows results for two elastically similar complete contacts with the material properties of the steel-steel case shown in Table 1. Note that $\xi_{\text {norm }}=$ 0.1450 and 0.0648 for the $90^{\circ}$ and $120^{\circ}$ punches, respectively.

An interesting implication of Figs. 10 and 11 is that although $\lambda_{1}$ and $\lambda_{2}$ are slightly modified based on the geometric and material properties of each particular problem, the results remain similar in all of these plots. In fact, the largest change in the results (for cases with the same edge angle) is between the uncoupled $90^{\circ}$ contact and the $90^{\circ}$ complete contact for which $\lambda_{2}$ changes by slightly more than $10 \%$. Moreover, $\lambda_{1}$ never changes by more than $5 \%$ from the uncoupled case, which indicates that $\lambda_{1}$ and $\lambda_{2}$ are

\footnotetext{
${ }^{10}$ See Appendix A for the definition of $v_{a}$ and $v_{b}$.
} 


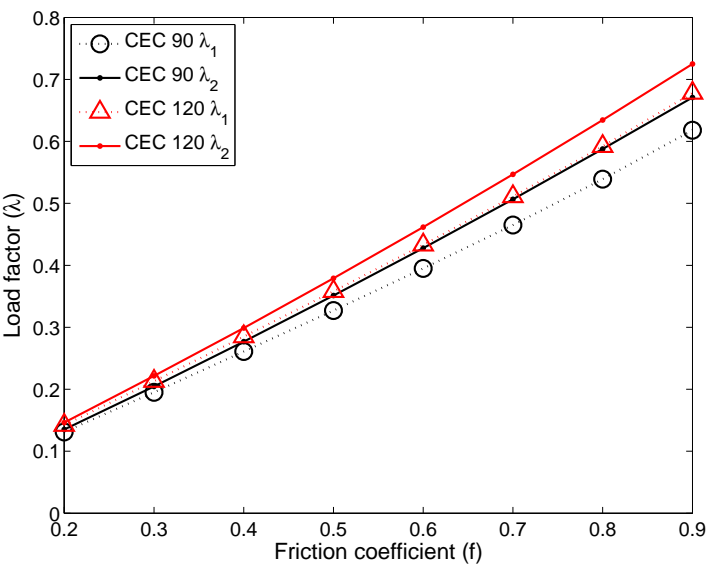

(a)

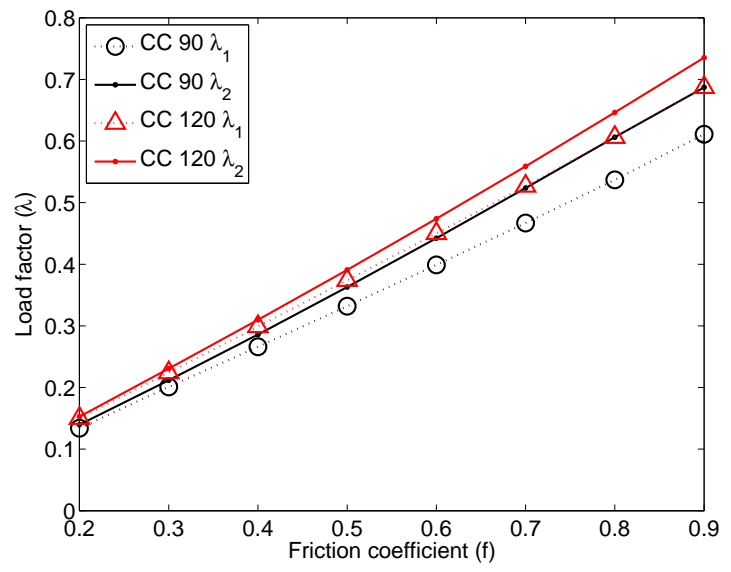

(b)

Figure 11: $\lambda_{1}$ and $\lambda_{2}$ plotted against friction coefficient, $f$, for (a) common-edged contacts with $\alpha=$ $0.4815, \beta=0.1376$ and (b) elastically similar complete contacts.

insensitive to elastic and geometric dissimilarity for these cases.

Figure 11 also illustrates that the size of the conditional region, $\Gamma$, increases with increasing friction. However, the coupling metrics defined in Eq. (4) do not take friction into account and depend solely on the contact's geometry. Thus, it may be more appropriate to correlate $\Gamma$ against the product of the coupling metric, $\xi$, and the friction coefficient, $f$, rather than against either of these parameters alone.

$\Gamma$ is plotted against $\xi_{\text {norm }} f$ in Fig. 12(a) and against $\xi_{\text {eig }} f$ in Fig. 12(b) for numerous contact geometries and material combinations. The material properties that were used for these contacts are given in Table 1 . These results demonstrate that $\Gamma$ is well predicted by $\xi f$; in fact, $\Gamma$ happens to be approximately equal to $\xi f$ for the cyclic shear loading considered here. However, $\xi_{\text {norm }} f$ appears to be a slightly better predictor of $\Gamma$ than $\xi_{\text {eig }} f$ since the data is grouped into a narrower band when it is plotted against $\xi_{\text {norm }} f$, though it is not clear why this is the case. It should be emphasized that both plots in Fig. 12 include results from very different contact problems: data from complete and common-edged contacts with edge angles of $90^{\circ}$ and $120^{\circ}$ are displayed together. The only parameter that was held constant is the loading regime, yet $\Gamma$ is well predicted by $\xi f$ for all these problems.

\subsection{Coupled contacts under cyclic bulk loading}

This section extends the analysis performed above to the cyclic bulk loading regime shown in Fig. 9(b). For this loading regime, $P$ is applied exactly as in the previous simulations, and then a bulk load, $\sigma$, is applied to the half-plane, which is cycled within the range $\left[\sigma_{\min }, \sigma_{\max }\right]$, where $\sigma_{\min }=-\sigma_{\max }$, until a steady-state response is reached. For this loading regime, the load factor is defined as $\lambda=\sigma_{\max } / P$. 


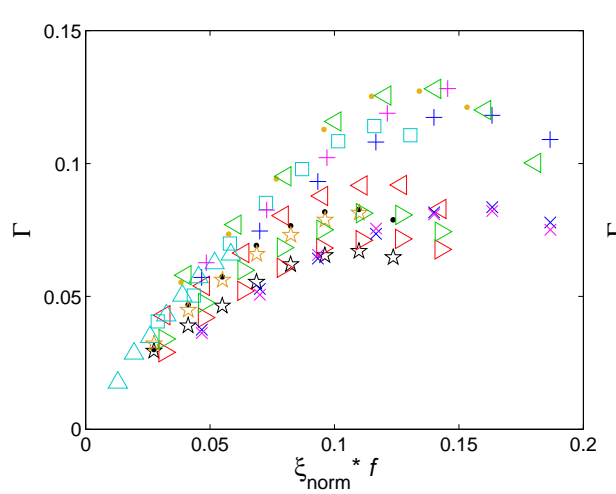

(a)

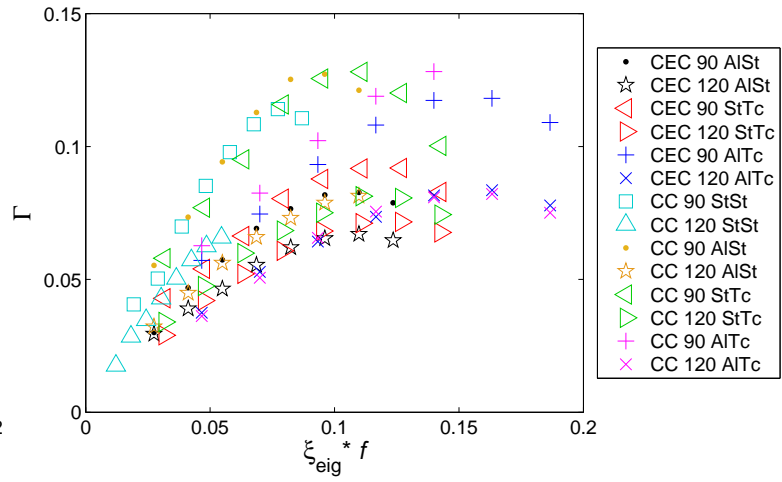

(b)

Figure 12: $\Gamma$ plotted against (a) $\xi_{\text {norm }} f$ and (b) $\xi_{\text {eig }} f$ for several complete (CC) and common-edged (CEC) contacts.

Note that the following analysis is restricted to complete contacts since it is unlikely that a common-edged contact would be subjected to a bulk load. As before, only the $90^{\circ}$ and $120^{\circ}$ contact angles are examined.

When estimating $\lambda_{1}$, a null displacement distribution was used to initialize the algorithm, and only one update of the displacement shape was performed. However, in this case, a sinusoidal displacement distribution is used, such that $v_{b}=\sin (2 \pi x / a)$. This displacement distribution was chosen because many of the optimal displacement distributions resulting from the calculation of $\lambda_{2}$ resemble sinusoidal functions for this cyclic bulk loading regime.

Figure 13 shows $\Gamma$ plotted against $\xi f$ for $90^{\circ}$ and $120^{\circ}$ complete contacts with numerous different material combinations subjected to the cyclic bulk loading regime shown in Figure 9(b). Note that the results from Fig. 12 are also included in these plots for comparison. Figure 13 illustrates that the conditional region is significantly larger when $\phi=90^{\circ}$ than when $\phi=120^{\circ}$. Moreover, although the data for the $120^{\circ}$ contact angle can be seen to lie along the same curve as the previous data for cyclic shear loading, this is probably just a coincidence. Indeed, it is reasonable to expect that if numerous other values of $\phi$ were studied, the data would fall into groups for each contact angle but that the initial-condition sensitivity would increase smoothly as $\phi$ decreases. Thus, $\phi=120^{\circ}$ probably just so happens to have a similar sensitivity to initial conditions as the shear loading case, but smaller angles would be more sensitive to initial conditions, and larger angles would be less sensitive to initial conditions. Some evidence for this speculation is that all of the data for the $90^{\circ}$ contact angle (including all the different material combinations) falls into a single group even though it does not lie on the same curve as the other data. 


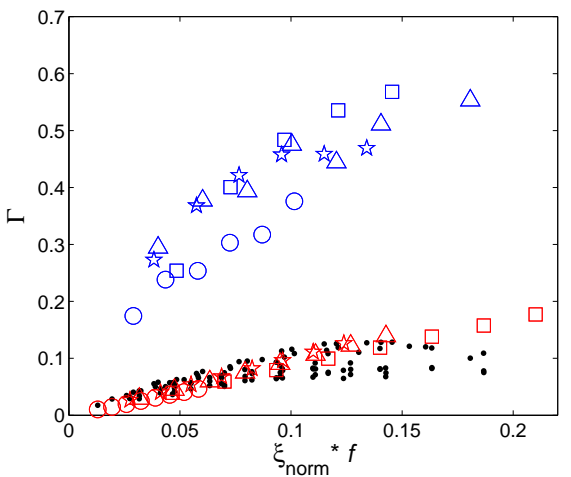

(a)

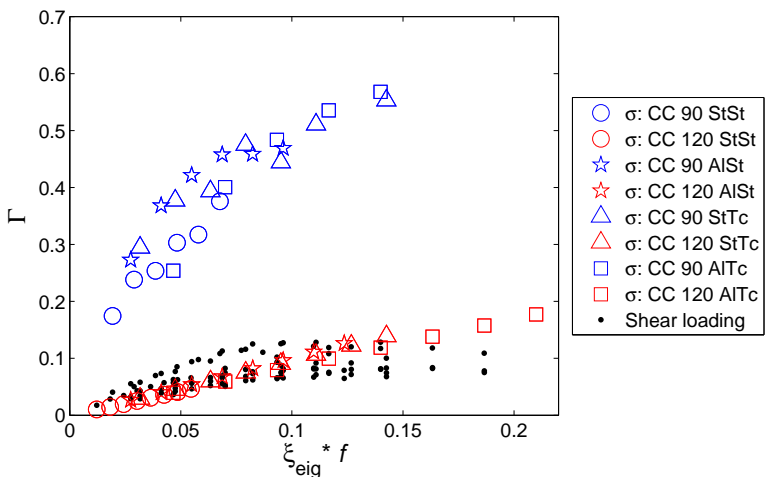

(b)

Figure 13: $\Gamma$ plotted against (a) $\xi_{\text {norm }} f$ and (b) $\xi_{\text {eig }} f$ for several complete (CC) and common-edged (CEC) contacts.

\subsection{Comparison of bulk and shear loading}

A question that immediately arises from Fig. 13 is: why are all of the results for $90^{\circ}$ complete contacts under cyclic bulk loading so much more sensitive to initial conditions than all of the other contact configurations? One hypothesis might be that this discrepancy is due to separation occurring at the contact edges. However, examination of the data reveals that separation does not occur in any of the simulations (for this or any other case).

To investigate this discrepancy in more detail, Fig. 14 shows the evolution of the normalized slip displacement, $v(x) / a$, along the contact interface, $x / a$, for three contact problems. All three contacts are between elastically similar materials, have an edge angle of $90^{\circ}$, use a friction coefficient of 0.7 , and use a null initial displacement condition. Figure 14(a), (b), and (c) show results for a common-edged contact under cyclic shear, a complete contact under cyclic shear, and a complete contact under a cyclic bulk load, respectively. The results shown in Fig. 14(a), (b), and (c) are performed with $\lambda=0.56,0.6$, and 2.5, respectively, and $\lambda_{2}=0.46,0.52$, and 1.96, respectively, for these three contact problems. Thus, all of these simulations are performed with $\lambda$ moderately above $\lambda_{2}$, resulting in cyclic slip in the steady state. Although these plots are for contacts with a $90^{\circ}$ edge angle, the contacts' qualitative responses do not change if the edge angle is changed to $120^{\circ}$.

Figure 14 shows both the transient phase of the contact's evolution and one cycle of its steady-state response. The most notable feature of this figure is the difference between the location of the cyclic slip zone for the bulk and shear loading cases. For both common-edged and complete contacts subjected to a cyclic shear load, the steadystate slip zone occurs at the center of the contact interface. Conversely, when a cyclic bulk load is applied, the steady-state slip zone is located at the edges of the interface. 


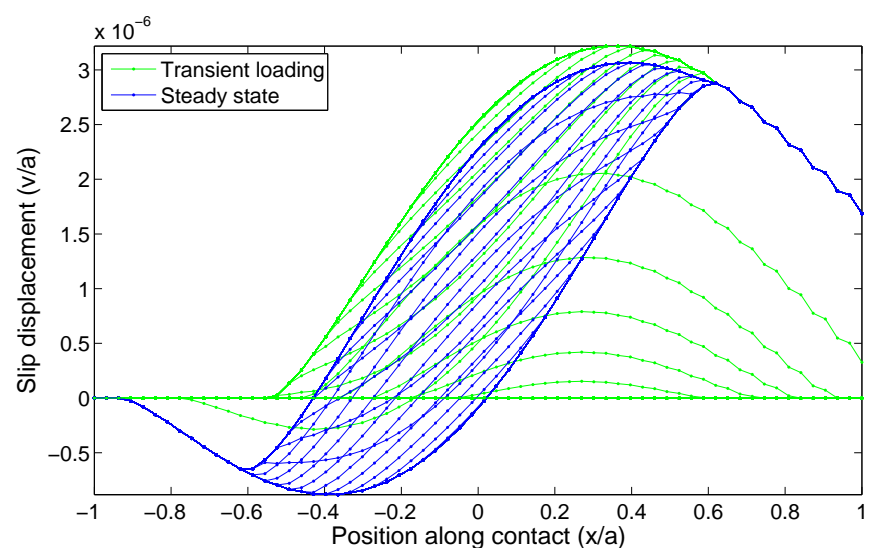

(a)

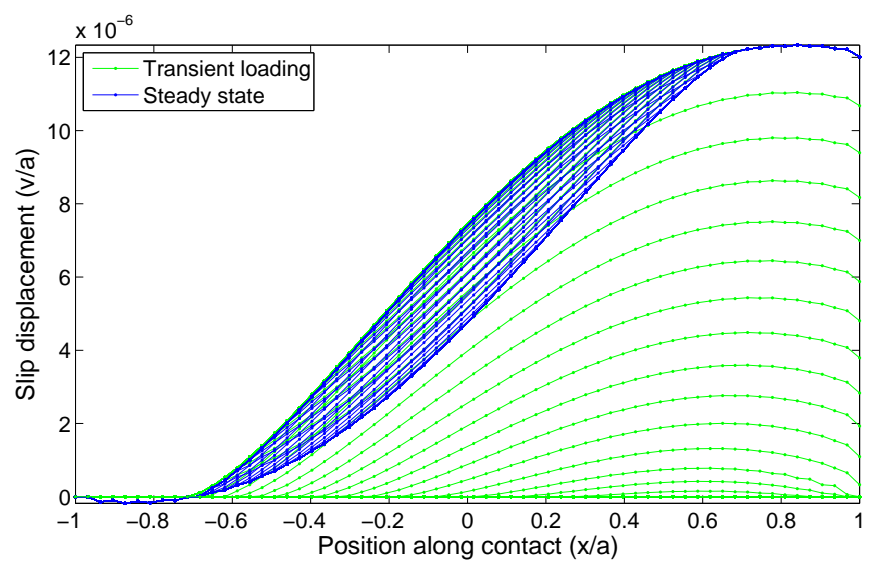

(b)

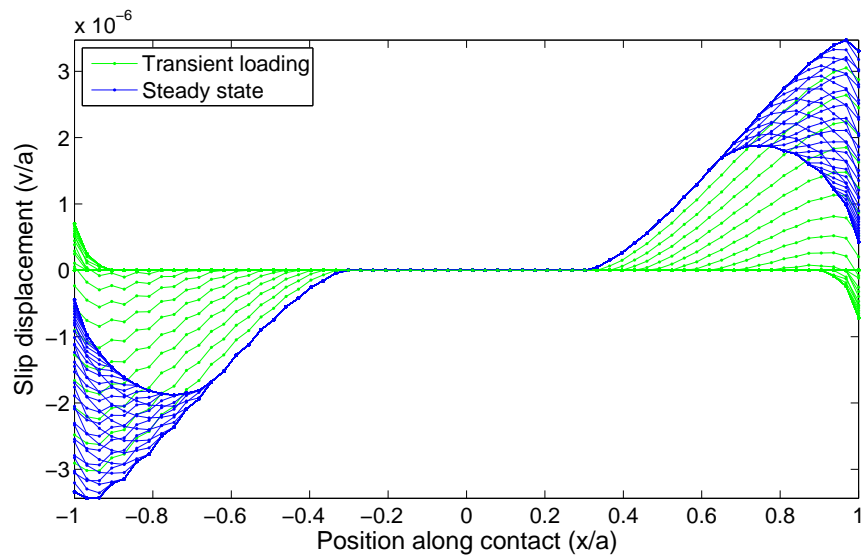

(c)

Figure 14: The evolution of the slip displacement, $v(x) / a$, plotted against normalized distance along the contact interface, $x / a$ for: (a) a $90^{\circ}$ common-edged contact under cyclic shear of $\lambda=0.56$, (b) a $90^{\circ}$ complete contact under cyclic shear of $\lambda=0.6$, and (c) a $90^{\circ}$ complete contact under a cyclic bulk load of $\lambda=2.5$. 
Thus, Fig. 14 explains why the cyclic shear problems are insensitive to the edge angle: the important slip zone occurs at the center of the interface where the influence of the contact-edge stress state is minimal. Conversely, the cyclic bulk load problems are highly sensitive to changing the edge angle because the steady-state slip zone is located at the edge of contact where the stress state is heavily influenced by the contact angle. Although the results are not shown in Fig. 14, varying the level of material dissimilarity or the friction coefficient does not modify the shape or location of the steady-state slip zone. Hence, this provides an explanation for why the coupling metric is able to predict a contact's response for any material combination and friction coefficient once the loading regime and contact angle are fixed.

These results suggest that the coupling metrics can be used to predict a contact's sensitivity to initial conditions when the steady-state slip zone remains in the same location. If the slip location changes, then the curves of $\Gamma$ vs. $\xi f$ must be recalibrated for those interface conditions. The results also suggest that changing the material combination or friction coefficient does not significantly modify the slip location, but changing the loading regime often does. Hence, results should be recomputed whenever the loading regime or contact geometry is modified.

\section{Discussion and conclusions}

The purpose of this paper is to develop a method for predicting how sensitive frictional contacts are to their initial conditions. This is important because the manufacturing process always introduces some degree of uncertainty into engineering assemblies, which is likely to manifest as unknown residual stresses or other interfacial conditions. Thus, it is essential for engineers to be able to predict how much a given structure's performance can be impacted by uncertainty of this form.

The approach taken in this paper is to investigate whether simple metrics of frictional coupling can be correlated against a contact's sensitivity to initial conditions. Since efficient methods already exist for calculating the shakedown limit, $\lambda_{2}$, knowledge of a contact's sensitivity to initial conditions can be used along with $\lambda_{2}$ to estimate $\lambda_{1}$. This information specifies the range of steady-state responses that a contact can exhibit over a wide range of loading conditions, which could give designers more confidence that a given structure will not behave in an unexpected way due to uncertainty associated with the way it is manufactured.

The first part of this paper focuses on examining the properties of the two coupling metrics proposed by Brake et al. [19] (i.e. $\xi_{\text {norm }}$ and $\xi_{\text {eig }}$ ). This is done in several steps in order to isolate the influence of coupling due to geometric dissimilarity and material dissimilarity. The results indicate that coupling due to material mismatch is dominated by Dundurs' parameter $\beta$, while Dundurs' parameter $\alpha$ has a much weaker effect. In addition, geometric mismatch has a stronger effect than $\alpha$ but a weaker effect than $\beta$. 
Subsequently, contacts with coupling arising from both material and geometric dissimilarity are examined, and the results indicate that coupling is dominated by geometric dissimilarity when $\beta$ is small, but material mismatch due to $\beta$ is the dominant source of coupling when $\beta \geqslant 0.2$. However, common engineering material combinations rarely result in $\beta>0.25$ (e.g. aluminum in contact with tungsten carbide gives $\beta=0.2338$ ), so geometric dissimilarity will be the dominant source of coupling in most practical contact problems.

In the second part of this paper, the relationship between the coupling metrics and a contact's sensitivity to initial conditions is investigated. To facilitate this process, the authors developed an algorithm for estimating $\lambda_{1}$, which is discussed in detail in $\S$ Appendix A. It was necessary to develop this algorithm because it is difficult to quantify a contact's sensitivity to initial conditions without knowledge of both $\lambda_{1}$ and $\lambda_{2}$. However, the only currently available technique for calculating $\lambda_{1}$ is prohibitively computationally expensive [16].

Thus, the algorithm presented in $\S$ Appendix A for estimating $\lambda_{1}$ is used along with the algorithm from [17] for calculating $\lambda_{2}$ to estimate the size of the conditional region, $\Gamma$, for numerous contact problems. First, some simple uncoupled contacts are examined to verify that the $\lambda_{1}$ estimation algorithm behaves as expected, i.e. that $\lambda_{1}=\lambda_{2}$ when $\xi=0$. Subsequently, $\Gamma$ is determined for numerous complete and common-edged contacts with various material combinations subjected to cyclic shear loading. Some of these results are plotted against the friction coefficient, $f$, which reveals that $\Gamma$ increases with increasing friction. These data are then plotted against $\xi f$, and a good correlation is obtained between $\xi f$ and $\Gamma$ for all of the contacts that are subjected to cyclic shear loading. Several $90^{\circ}$ and $120^{\circ}$ complete contacts with different material combinations are then subjected to cyclic bulk loading. The results show that the $90^{\circ}$ complete contacts are significantly more sensitive to initial conditions than $120^{\circ}$ complete contacts under cyclic bulk loading and all of the shear loading cases. This suggests that sensitivity to initial conditions increases with edge angle for cyclic bulk loading, whereas the edge angle does not influence shear-loaded contacts' sensitivity to initial conditions.

To examine this discrepancy, several plots of the of the transient evolution of the slip displacement are shown for shear loading and bulk loading cases, which reveal a key difference between these types of loading regimes: shear loading excites slip at the center of the interface, whereas bulk loading excites slip at the edges of the interface. The stress state in the slip zone is what determines whether shakedown occurs. Thus, these results show that when slip occurs at the center of the interface, the results are much less sensitive to contact geometry and edge angle. Conversely, when slip occurs at the edges of the interface, the contact-edge stress state strongly influences whether shakedown occurs, and $\Gamma$ is much more sensitive to changes in the contact-edge angle.

The implications of these results are that $\xi$ can be used to predict a contact's sens- 
itivity to initial conditions provided that the location where slip occurs remains similar. However, if the location of the slip zone changes significantly, the results need to be recalibrated for the new slip location. This entails estimating $\lambda_{1}$ using the algorithm from $\S$ Appendix A, calculating $\lambda_{2}$ using the approach in [17], and then estimating $\Gamma$ for a range of friction coefficients. This calibration process is computationally expensive, so future work should focus on developing coupling metrics that account for the location of the steady-state slip zone.

The reason re-calibration is necessary when the location of the slip zone changes is that these coupling metrics quantify coupling in an aggregate sense, and they are independent of the loading history. In order to remove the need for re-calibration, a coupling metric would need to be developed that takes the loading regime and contact behavior into account. It is not clear how to define such a metric, but it certainly must depend on more than just the $\boldsymbol{A}$ and $\boldsymbol{B}$ matrices. Thus, future work could investigate how to develop a more robust coupling metric.

\section{Acknowledgments}

The authors would like to thank Sean Topping for his help developing the subroutine described in Appendix A.2.

\section{Appendix A. Algorithm for estimating $\lambda_{1}$}

A high-level overview of the algorithm for obtaining an upper-bound estimate of $\lambda_{1}$ is shown in Fig. 15. Note that a prerequisite for using this algorithm is that the contact stiffness matrix, $\boldsymbol{K}$, and the loading vectors, $\boldsymbol{f}^{w}$, for all of the applied loads must already have been calculated as described in [20]. This algorithm begins by assuming a particular initial condition, $v_{0}=v_{a}$, which is often chosen to be the null case (i.e. $v_{0}=$ $0)$, though any initial condition can be used.

Notice that for any given combination of $v_{0}$ and $\lambda$, the steady-state response is unique (since the model is deterministic). Therefore, if $v_{0}$ and the loading regime are fixed, ${ }^{11}$ but $\lambda$ is allowed to vary, there is a single load factor, $\Lambda_{S D}$, that marks the boundary between the contact shaking down and the contact reaching a dissipative steady state. This is simply because once $v_{0}$ is fixed, there can only be a single steady-state solution for each load factor, and this solution must either be dissipative or non-dissipative (i.e. shaken down).

The next step in this algorithm is to perform a series of transient simulations to determine $\Lambda_{S D}$, which can be thought of as the shakedown limit for the current $v_{0}$.

\footnotetext{
${ }^{11}$ Fixing the loading regime is meant in the sense that the points at which the loads are applied and the directions in which they act are not modified. The only thing that is allowed to vary is the amplitude of these loads or, in other words, the load factor.
} 


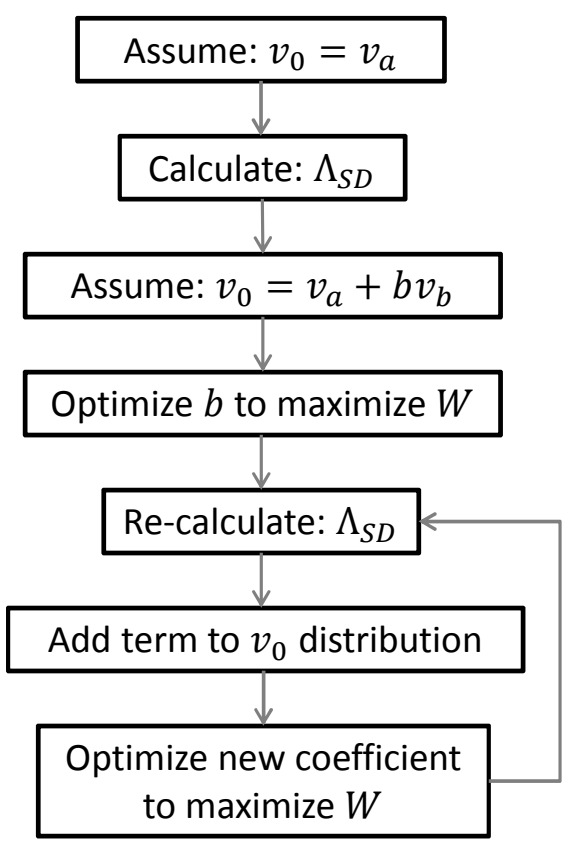

Figure A.15: Block diagram of the algorithm for estimating $\lambda_{1}$.

However, it must be emphasized that $\Lambda_{S D}$ generally is not equal to $\lambda_{1}$ or $\lambda_{2}$; it is simply the load factor that separates dissipative and non-dissipative steady-state responses for a particular initial condition. In contrast, $\lambda_{1}$ and $\lambda_{2}$ are properties of the system that do not depend on $v_{0}$. A description of the subroutine for calculating $\Lambda_{S D}$ is provided in $\S$ Appendix A.1.

Once $\Lambda_{S D}$ has been determined, it can be regarded as a first-order estimate of $\lambda_{1}$. In order to obtain a better estimate of $\lambda_{1}$, the initial displacement is updated with the aim of obtaining the initial condition that maximizes dissipation when $\lambda=\Lambda_{S D}$. To do this, $v_{0}$ is assumed to be given by the sum of the previous initial displacement, $v_{a}$, and a second term, $b v_{b}$, where $v_{b}$ is a known displacement distribution, and $b$ is a dimensionless scalar multiple that controls its magnitude. Note that $v_{b}$ can be any displacement distribution, but it must be selected manually by the user. ${ }^{12}$ Some of the distributions that the authors examined include linear, sinusoidal, and random distributions. These simulations suggest that linear and low-frequency sinusoidal distributions have the largest influence on the contact's steady-state response. A discussion of the subroutine for optimizing $v_{0}$ to maximize steady-state dissipation is provided $\S$ Appendix A.2.

Once $v_{0}$ is updated, the next step is to recalculate $\Lambda_{S D}$ for this updated initial displacement condition, which provides an improved estimate of $\lambda_{1}$. As shown in Fig. 15,

\footnotetext{
${ }^{12}$ See $\S$ Appendix A.3.3 for some approaches for choosing the initial displacement shapes.
} 

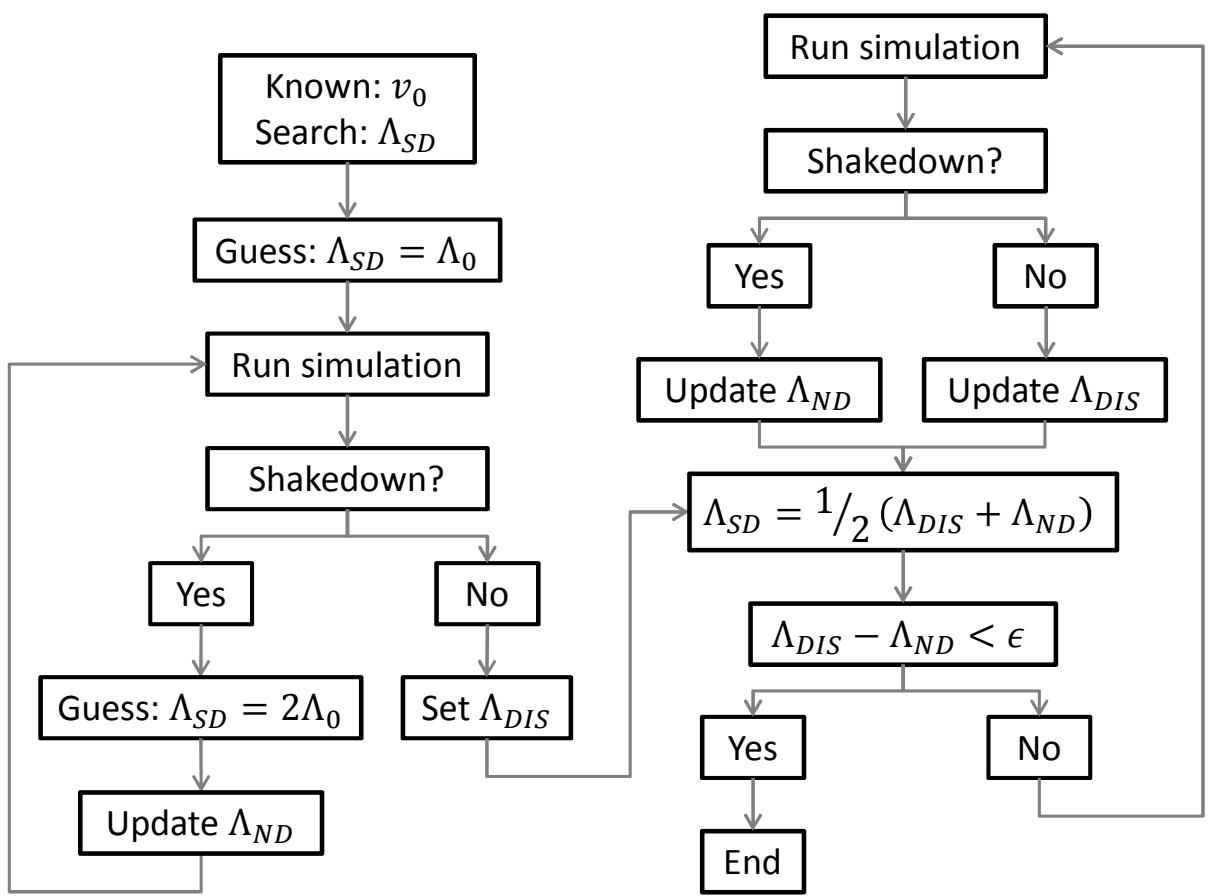

Figure A.16: Block diagram of the subroutine for calculating $\Lambda_{S D}$.

the algorithm can then continue by adding more terms to $v_{0}$ and updating $\Lambda_{S D}$ to obtain successively better estimates of $\lambda_{1}$. This process of updating $v_{0}$ can then be repeated several times. Note that the authors found that only the first couple of updates of $v_{0}$ resulted in a noticeable change to $\Lambda_{S D}$, so $v_{0}$ is only updated once for the results presented in this paper. Also note that there generally are multiple $v_{0}$ distributions that will cause the algorithm to find the correct value of $\lambda_{1}$.

\section{Appendix A.1. Calculation of $\Lambda_{S D}$}

A block diagram of the subroutine for calculating $\Lambda_{S D}$ is shown Fig. 16. The first block in this diagram simply states that $v_{0}$ is known (and is held constant), whereas $\Lambda_{S D}$ is unknown. Thus, the algorithm searches for $\Lambda_{S D}$ by running several transient simulations with different $\lambda$ (but with the same $v_{0}$ ). The first step in this subroutine is to make an initial guess of $\Lambda_{S D}$, which is denoted $\Lambda_{0}$. Using a good guess for $\Lambda_{0}$ speeds up the runtime for this subroutine, but the algorithm converges provided only that $\Lambda_{0} \neq 0$. Note that it is often convenient to set $\Lambda_{0}=\lambda_{2}$ since it can be calculated quickly using the algorithm described by Flicek et al. [17] and tends to be a relatively good starting point.

A transient simulation is then performed with $\lambda=\Lambda_{0}$ and $v_{0}=v_{a}$, which is run until a steady-state response is achieved. The definition of when a steady state is achieved 
is somewhat arbitrary, but the conditions used in the authors' implementation of the algorithm are the same as those used by Flicek et al. [17]. Specifically, it is assumed a steady state is reached when: (i) the dissipation per cycle, $W$, is similar in size to the smallest meaningful value of $W$ that can be represented with a double-precision floating-point number, or (ii) the change in $W$ from the previous load cycle is less than $0.01 \%$. Note that multiple algorithms can be used to perform the transient simulations required in this algorithm, but the authors used the Gauss-Seidel algorithm described by Ahn and Barber [10].

Once the simulation has completed, the algorithm checks whether the simulation shook down based on whether the dissipation is zero (within some small tolerance) in the final cycle of the simulation. If the simulation does not shake down, then the value of $\Lambda_{S D}$ from this simulation is recorded as $\Lambda_{D I S}$, which is the lowest $\lambda$ that has resulted in a dissipative steady-state response for the current initial condition. Conversely, if the simulation shook down during this initial simulation, the value of $\Lambda_{S D}$ from this simulation is recorded as $\Lambda_{N D}$, which is the highest $\lambda$ that has resulted in shakedown for the current $v_{0}$. The initial guess for $\Lambda_{S D}$ is then doubled, and this process is repeated until the simulation does not shake down, and a value for $\Lambda_{D I S}$ is obtained. Note that at the onset of this subroutine, $\Lambda_{N D}$ is set to a default value of zero, so only $\Lambda_{S D}$ must be determined before proceeding to the next phase of the subroutine.

Once a value for $\Lambda_{D I S}$ is obtained, an updated guess for $\Lambda_{S D}$ is made based on the bisection method: that is, $\Lambda_{S D}=0.5\left(\Lambda_{D I S}+\Lambda_{N D}\right)$. The accuracy of this new guess of $\Lambda_{S D}$ is then estimated by looking at the difference between $\Lambda_{D I S}$ and $\Lambda_{N D}$. If the accuracy is within some user-defined tolerance $\epsilon$, then the subroutine terminates, and the most recent estimate of $\Lambda_{S D}$ is returned. Conversely, if the accuracy is not within the tolerance, then another simulation is performed with the updated guess for $\Lambda_{S D}$. If this subsequent simulation shakes down, $\Lambda_{N D}$ is updated, and if it fails to shake down, $\Lambda_{D I S}$ is updated. The guess for $\Lambda_{S D}$ is then recalculated, and this process continues until $\Lambda_{S D}$ is within the user-specified tolerance, $\epsilon$.

\section{Appendix A.2. Initial condition optimization}

A block diagram of the subroutine for updating $v_{0}$ is shown in Fig. 17. The first block in this diagram states that $\Lambda_{S D}, v_{a}$, and $v_{b}$ are all known quantities at the onset of this subroutine, and the objective is to optimize $v_{0}$. Note that $\Lambda_{S D}$ is known because it is determined by the previously described subroutine, and $v_{a}$ and $v_{b}$ are displacement distributions that must be specified by the user.

The first step in this subroutine is to assume an initial displacement of the form $v_{0}=v_{a}+b v_{b}$, where $b$ controls the amplitude of the displacement shape $v_{b}$. The next step is to specify the size of the search space by defining the interval $\left[b_{\min }, b_{\max }\right]$. Since each simulation that is performed is often quite computationally expensive, it is desirable to run as few simulations as possible when searching for an updated displacement shape. One way to achieve this is first to recognize that there is usually a monotonic relationship 


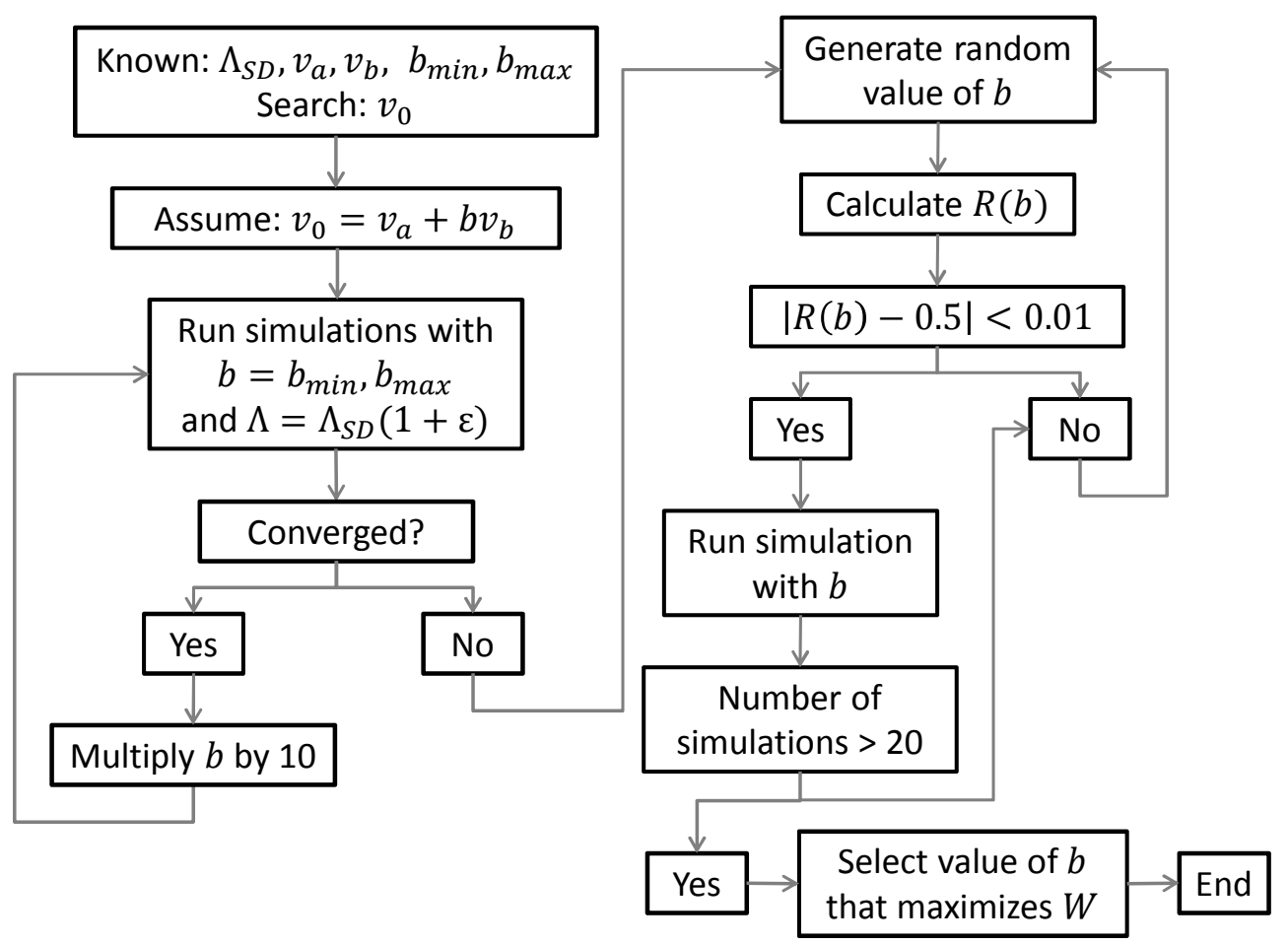

Figure A.17: Block diagram of the subroutine for optimizing $v_{0}$ to maximize dissipation.

between the amplitude of the initial displacement and the energy that is dissipated by the contact. Therefore, the initial condition that has the largest impact on steady-state dissipation is likely to be an extreme value. However, if a sufficiently large initial displacement condition is applied to the contact, the simulation fails for numerical reasons. $^{13}$

Therefore, one approach for reducing the number of simulations is simply to search

\footnotetext{
${ }^{13}$ Specifically, the algorithm for calculating the evolutionary contact solution uses the GaussSeidel iterative technique. This algorithm begins by assuming a particular state for each node (i.e. stick, forward slip, backward slip, or separation). It then checks each node one at a time to see if the normal and shear forces at that node violate the contact or friction conditions (i.e. the contact pressure cannot be tensile, and $q_{i}<f p_{i}$ ). It then updates the assumed state based on the nature of the violation (e.g. if the contact pressure is tensile, it assumes the node has separated). The algorithm performs this state updating iteratively until none of the assumed states violate the relevant conditions. However, because it checks the nodes one at a time, if too large a change in the displacement state occurs in a single timestep, it is possible, for example, for a node to be assumed to be stuck but then violate the contact conditions, implying its separated. However, when that same node is assumed to have separated, it may violate the conditions implied by the separated state, implying that it is stuck. Thus, the algorithm can end up in an infinite loop where it never guesses all of the node's states correctly.
} 
for the displacement amplitude above which the simulation fails for numerical reasons. A response variable, $R$, can be defined, such that $R=0$ for simulations that fail, and $R=1$ for simulations complete successfully. Simulations are then performed for $b_{\max }$ and $b_{\min }$ when $\lambda=\Lambda_{S D}(1+\epsilon)$, where $\epsilon$ is a small number, e.g. $0.01 .{ }^{14}$ If either of these simulations do not fail, the value of $b_{\max }$ or $b_{\min }$ is increased by an order of magnitude and the simulation is run again. This process is repeated until the simulations for both $b_{\max }$ and $b_{\min }$ fail. Note that $b=0$ is known to complete successfully since this is the final simulation that is performed during the search for $\Lambda_{S D}$.

The most dissipative initial condition must now result from a value of $b$ within the interval $\left[b_{\min }, b_{\max }\right]$. However, not all values of $b$ within this range result in simulations that complete successfully. Therefore, this subroutine uses a random sampling technique to find the upper and lower limits of $b$ that result in simulations that complete successfully. To minimize the number of simulations that are preformed, before performing each simulation, all of the data from the previously run simulations are used to predict how likely the current value of $b$ is to complete successfully. This is done by calculating a weighted average of the responses of the previously run simulations, where the weighting for each term is determined by its distance from the current value of $b$, such that closer points have a larger impact on the predicted response [25, 26]. One expression for doing this is given by

$$
R(b)=\frac{\sum_{i=1}^{M} \frac{R_{i}}{\left(b_{i}-b\right)^{2}}}{\sum_{i=1}^{M} \frac{1}{\left(b_{i}-b\right)^{2}}},
$$

where $M$ is the number of simulations that are performed, $R_{i}$ is the response of the $i$ th simulation, $b_{i}$ is the displacement amplitude for the $i$ th simulation, $b$ is the current displacement amplitude, and $R(b)$ is the predicted response for the current value of $b$. Note that $R_{i}$ is an integer in the set $\{0,1\}$, whereas $R(b)$ is a real number in the interval $[0,1]$. Also note that the exponent of 2 on the term $\left(b_{i}-b\right)$ could be varied within the interval $(0, \infty)$. Increasing this exponent biases the calculation to give a higher weighting to the points that are closest to $b$.

A value of $R(b)$ that is close to 0 means that the simulation is likely to fail, whereas a value close to 1 suggests the simulation is likely to complete successfully. Conversely, a value of $R(b)$ that is close to 0.5 suggests that the current value of $b$ is near a boundary

\footnotetext{
${ }^{14}$ Note that these simulations could also be performed with $\lambda=\Lambda_{S D}$, but it tends to be easier to determine the effect that varying $b$ has on the system's steady-state response when the contact just barely fails to shake down when $b=0$ (which is achieved by running simulations at a load factor slightly greater than $\Lambda_{S D}$ ). The inclusion of $\epsilon$ is also essential if this algorithm is modified slightly and used to estimate $\lambda_{2}$.
} 
between the simulation failing and completing successfully. Thus, this subroutine randomly generates values of $b$ within the interval $\left[b_{\min }, b_{\max }\right]$ until a value of $b$ is found that results in a predicted response that is within some small tolerance of 0.5 . A simulation is then performed for this value of $b$.

This process is then repeated multiple times. Note that the predicted value of $R(b)$ is based on increasing amounts of data in each successive iteration. In this way, simulations are only performed for values of $b$ that are close to the boundary between the simulation failing and completing successfully, which are likely to have the largest influence on dissipation, $W$. This boundary can be defined quite well by repeating this process 15-20 times. Once several iterations are performed, the value of $b$ that results in the maximum dissipation in this set of simulations is returned to the main algorithm.

\section{Appendix A.3. Algorithm accuracy}

Given that this algorithm only provides an upper-bound estimate of $\lambda_{1}$ and not an exact solution, it is important to be able to assess the degree of error that is likely to be associated with its use. Perhaps the most obvious way to quantify this algorithm's accuracy is to select a sufficiently small contact problem for which $\lambda_{1}$ can be calculated exactly using the approach presented in [16]. However, one potential issue with this approach is that there may be important differences between small problems with 10-20 contact nodes and larger problems with 50-500 contact nodes. Indeed, the authors found some evidence for this assertion when conducting a converge study to determine the mesh resolution to use for the simulations in this paper.

\section{Appendix A.3.1. Mesh sensitivity of the steady state solution}

The authors conducted several convergence studies using meshes with $4,8,16$, 32, 64, 128, 256, and 512 contact nodes to determine the mesh refinement required to accurately capture the micro-slip behavior along the interface. This was done by selecting a few example problems and determining the level of mesh refinement above which effectively identical results are obtained. For example, an elastically similar complete contact with a $90^{\circ}$ edge angle, such as that shown in Fig. 6, was subjected to the cyclic bulk loading shown in Fig. 9(b) until a steady state was reached with $\lambda=2$ and $f=0.7$. The results are plotted in Fig. 18, which shows the normalized steady-state slip displacement, $v / a$, plotted against the normalized position along the interface, $x / a$, for the 8 mesh refinements listed above.

Fig. 18 illustrates that significantly different contact behavior is observed for meshes with fewer than 64 nodes. On the basis of these results, the authors chose to use 128 contact nodes in $\S 3$ when assessing the coupling metrics and 64 contact nodes in $\S 4$ for performing the transient simulations to assess the coupling metric's usefulness. The authors chose to use 128 contact nodes in $\S 3$ since calculating the coupling metrics is quite computationally inexpensive, so the price of using 


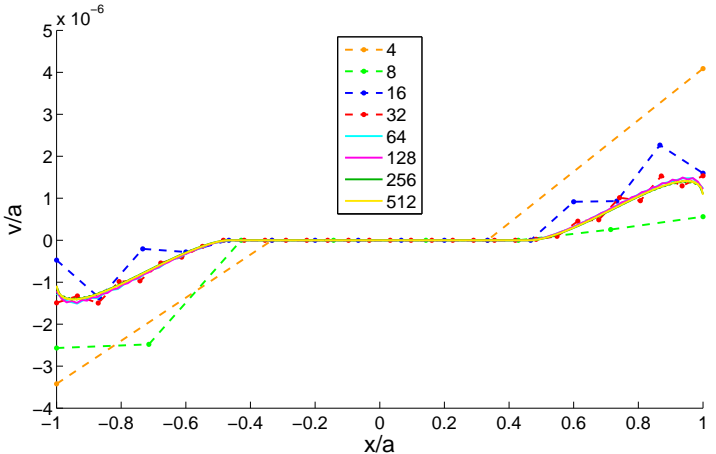

(a)

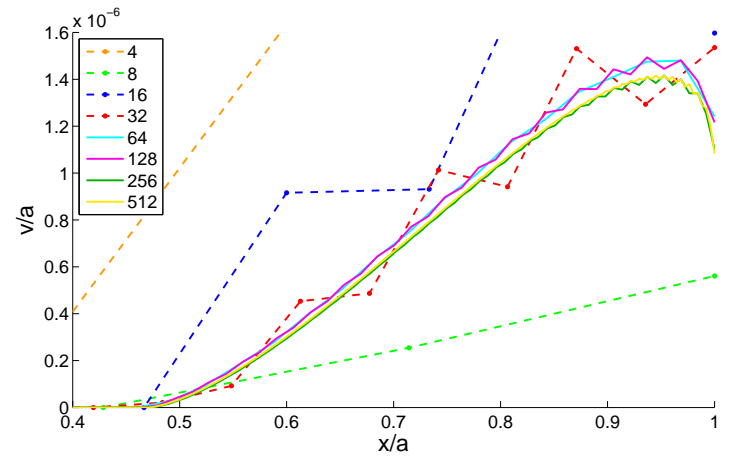

(b)

Figure A.18: Mesh sensitivity of the steady-state slip displacement, $v / a$ : (a) shown full scale and (b) a zoomed-in view of the contact edge.

slightly more refined models was quite low in this case. Conversely, $\S 4$ required a large number of transient simulations to be performed, which are much more computationally expensive, so the lowest possible level of refinement that provides converged results was selected.

\section{Appendix A.3.2. Estimating algorithm accuracy}

The accuracy of the algorithm presented in this section is very likely to problem dependent. Thus, the discrepancy in the results between the small models (i.e. 432 contact nodes) and the converged models (i.e. 64 or more contact nodes) casts some doubt on whether the accuracy of the algorithm's estimate of $\lambda_{1}$ for the small models would be similar to that for the converged models. Indeed, it's likely that this algorithm would be more accurate for the smaller models than for converged models since the number of possible $v_{0}$ distributions increases with the number of contact nodes. However, instead of calculating $\lambda_{1}$ exactly for a small model using the algorithm from [16], an alternative approach for estimating this algorithm's accuracy can be used.

Notice that although the algorithm in its current form is designed to estimate $\lambda_{1}$, it is trivially simple to modify it to search for $\lambda_{2}$ instead. To do this, the subroutine described in $\S$ Appendix A.2 simply needs to return the value of $b$ that minimizes dissipation instead of that which maximizes it. With this simple change, the algorithm can be used to find $\lambda_{2}$. Since this change to the algorithm is so minor, the algorithm's accuracy is likely to be similar when searching for $\lambda_{1}$ or $\lambda_{2}$. Thus, the algorithm's accuracy can be estimated on the actual problem of interest by having it search for $\lambda_{2}$ and comparing its result to the exact value of $\lambda_{2}$ that's been calculated using the method described in [17].

The authors assessed the algorithm's accuracy in this way using two complete 


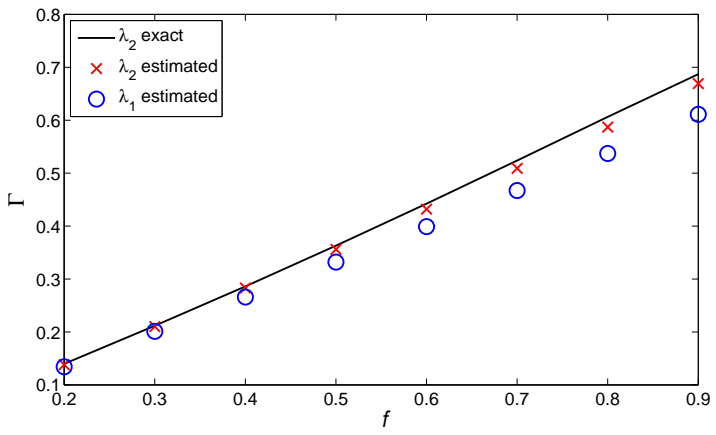

(a)

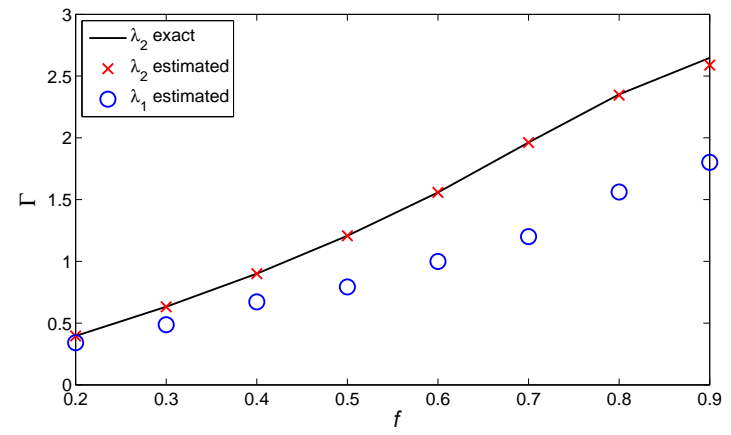

(b)

Figure A.19: Accuracy of the $\lambda_{2}$ estimation algorithm compared to the true value of $\lambda_{2}$ calculated as in [17] for elastically similar complete contacts subjected to: (a) cyclic shear loading and (b) cyclic bulk loading.

contact problems with $\phi=90^{\circ}$. The material properties of the bodies are given by the Steel-Steel case listed in Table 1. The authors subjected one contact to the cyclic shear loading shown in Fig. 9(a) and the other to the cyclic bulk loading shown in Fig. 9(b). The results are shown in Fig. 19, which shows the exact value of $\lambda_{2}$ calculated using the method in [17], the estimate of $\lambda_{2}$ using the modified algorithm discussed in this section, and the estimate of $\lambda_{1}$ using the original algorithm (using the same displacement shape that was used to search for $\lambda_{2}$ ). Note that a null initial $v_{0}$ was used (i.e. $v_{a}=0$ ) followed by a single $v_{0}$ update, where the updated displacement shape (i.e. $v_{b}$ ) was sinusoidal for both the shear loading and bulk loading.

Figure 19 illustrates that the algorithm's accuracy is indeed problem dependent: The algorithm is moderately more accurate for the bulk-loaded contact shown in Fig. 19(b) than for the shear-loaded contact shown in Fig. 19(a). However, the estimate of $\lambda_{2}$ is within $5 \%$ of the true value in all cases and the error is generally $2 \%$ or less. Thus, this suggests that provided that a satisfactory displacement shape is selected, the algorithm is likely to be quite accurate. However, the burden is on the engineer to perform a preliminary study to assess which displacement shape(s) to use.

\section{Appendix A.3.3. Selecting $v_{0}$ distributions}

A fairly simple way to select the initial displacement distributions is to: (i) calculate $\lambda_{2}$ using the method in [17] and examine the optimal displacement shape, and (ii) run a transient simulation until the steady state is reached and examine the steady-state displacement distribution. The engineer can then test the algorithm with a few $v_{0}$ shapes that resemble the two displacement distributions from this study and use the distribution that gives the lowest estimate for $\lambda_{1}$. Of course, the 


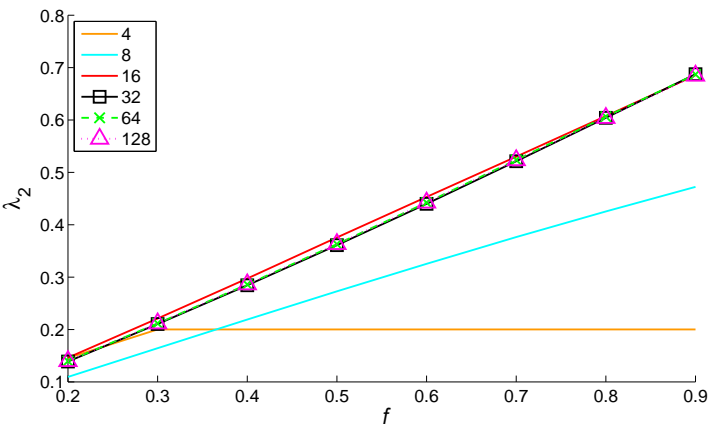

(a)

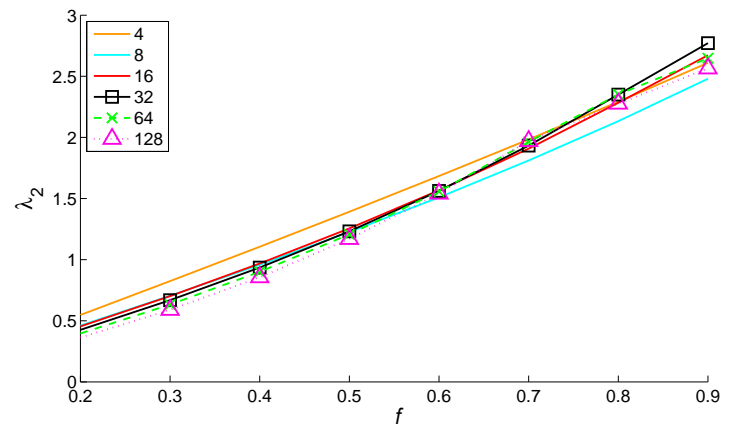

(b)

Figure A.20: Mesh sensitivity of the true value of $\lambda_{2}$ calculated as in [17] for an elastically similar complete contact with $\phi=90^{\circ}$ subjected to (a) cyclic shear loading and (b) cyclic bulk loading.

engineer could also simply use the exact displacement distributions resulting from one of these calculations.

Conversely, the engineer may not actually be interested in the true value of $\lambda_{1}$ and may instead be concerned with the lowest $\lambda$ for which shakedown may fail to occur for realistic initial displacement distributions. In this event, the $\lambda_{1}$ estimation algorithm could be restricted to whatever displacement distribution the engineer deems appropriate. For example, the algorithm could be restricted to linear distributions since these can be installed simply by applying a bulk tension to a specimen prior forming the contact, whereas it is much less obvious how a sinusoidal distribution could be installed in practice. However, the engineer must keep in mind that any restrictions on the $v_{0}$ distributions that can be used will result in a less conservative estimate of $\lambda_{1}$ and hence will underestimate the contacts sensitivity to initial conditions.

\section{Appendix A.3.4. Mesh sensitivity of shakedown limits}

One other question regarding the accuracy of the overall approach for calculating $\Gamma$ that is used in $\S 4$ is: How sensitive are the calculated shakedown limits (i.e. $\lambda_{1}$ and $\lambda_{2}$ ) to the mesh that is used? To provide at least a partial answer to this question, the authors used the approach in [17] to calculate $\lambda_{2}$ for example problems meshed with $4,8,16,32,64$, and 128 contact nodes.

Figure 20 shows these results plotted against friction coefficient, $f$, for an elastically similar complete contact with $\phi=90^{\circ}$ subjected to (a) cyclic shear loading and (b) cyclic bulk loading. The results suggest that $\lambda_{2}$ actually converges faster than the steady state displacement distribution, which is shown in Fig. 18. For both the shear and the bulk loading cases, $\lambda_{2}$ begins to converge with 16 contact nodes, and the results above 32 contacts nodes are effectively identical. However, the error associated with the 4 and 8 contact node models is significantly greater under 
cyclic shear loading than cyclic bulk loading.

These results suggest that provided that the mesh has been refined enough to provide converged slip displacements, $\lambda_{2}$ will also have converged. Although a similar mesh sensitivity study has not been performed on the $\lambda_{1}$ estimation algorithm, it is expected that it too will provide converged results provided that the slip displacements have converged. Thus, the estimates of $\Gamma$ presented in $\S 4$ are unlikely to change if a more refined mesh were used. However, if a coarser mesh were used, the results probably would change due to the model not accurately capturing the micro-slip occurring along the contact interface.

\section{References}

[1] J. Abad, J.M. Franco, R. Celorrio, and L. Lezáun. Design of experiments and energy dissipation analysis for a contact mechanics 3D model of frictional bolted lap joints. Adv. Eng. Softw., 45(1):42-53, 2012. doi: 10.1016/j.advengsoft.2011.09.021.

[2] S.S. Law, Z.M. Wu, and S.L. Chan. Analytical model of a slotted bolted connection element and its behaviour under dynamic load. J. Sound. Vib., 292(3): 777-787, 2006. doi: 10.1016/j.jsv.2005.09.028.

[3] R. Rajasekaran and D. Nowell. Fretting fatigue in dovetail blade roots: Experiment and analysis. Tribol. Int., 39:1277-1285, 2006. doi: 10.1016/j.triboint.2006.02.044.

[4] N.S. Xi, P.D. Zhong, H.Q. Huang, H. Yan, and C.H. Tao. Failure investigation of blade and disk in first stage compressor. Eng. Fail. Anal., 7(6):385-392, 2000. doi: 10.1016/S1350-6307(99)00045-X.

[5] L. Limmer, D. Nowell, and D.A. Hills. A combined testing and modelling approach to the prediction of the fretting fatigue performance of splined shafts. $P$. I. Mech. Eng. G-J. Aer., 215(2):105-112, 2001. doi: 10.1243/0954410011531808.

[6] N. Banerjee and D.A. Hills. Analysis of stick-slip and contact-edge behaviour in a simplified fretting fatigue test. J. Strain Anal. Eng., 41(3):183-192, 2006. doi: 10.1243/03093247JSA83.

[7] T.N. Farris, M.P. Szolwinski, and G. Harish. Fretting in aerospace structures and materials. In D.W. Hoeppner, V. Chandrasekaran, C.B. Elliott (Eds.), Fretting Fatigue: Current Technology and Practice, ASTM STP, volume 1367, pages 523537. ASTM, West Conshohocken, PA, 2000.

[8] L. Gaul and R. Nitsche. The role of friction in mechanical joints. Appl. Mech. Rev., 54(2):93-106, March 2001. doi: 10.1115/1.3097294. 
[9] M. R. W. Brake, editor. The Mechanics of Jointed Structures: Recent Research and Open Challenges for Developing Predictive Models for Structural Dynamics, to be published in 2017. Springer.

[10] Y.J. Ahn and J.R. Barber. Response of frictional receding contact problems to cyclic loading. Int. J. Mech. Sci., 50(10-11):1519-1525, October-November 2008. doi: 10.1016/j.ijmecsci.2008.08.003.

[11] J.H. Kim and Y.H. Jang. Frictional hertzian contact problems under cyclic loading using static reduction. Int. J. Solids. Struct., 51(1):252-258, 2014. doi: 10.1016/j.ijsolstr.2013.09.028.

[12] J.R. Barber, M. Davies, and D.A. Hills. Frictional elastic contact with periodic loading. Int. J. Solids. Struct., 48(13):2041-2047, 2011. doi: 10.1016/j.ijsolstr.2011.03.008.

[13] Y.H. Jang and J.R. Barber. Frictional energy dissipation in materials containing cracks. J. Mech. Phys. Solids, 59(3):583-594, 2011. doi: 10.1016/j.jmps.2010.12.010.

[14] A. Klarbring, M. Ciavarella, and J.R. Barber. Shakedown in elastic contact problems with Coulomb friction. Int. J. Solids. Struct., 44(25-26):8355-8365, December 2007. doi: 10.1016/j.ijsolstr.2007.06.013.

[15] J.R. Barber, A. Klarbring, and M. Ciavarella. Shakedown in frictional contact problems for the continuum. C. R. Mec., 336(1-2):34-41, January-February 2008. doi: $10.1016 /$ j.crme.2007.10.013.

[16] Y.J. Ahn, E. Bertocchi, and J.R. Barber. Shakedown of coupled two-dimensional discrete frictional systems. J. Mech. Phys. Solids, 56(12):3433-3440, December 2008. doi: 10.1016/j.jmps.2008.09.003.

[17] R.C. Flicek, D.A. Hills, J.R. Barber, and D. Dini. Determination of the shakedown limit for large, discrete frictional systems. Eur. J. Mech. A-Solid, 49:242-250, January-February 2015. doi: 10.1016/j.euromechsol.2014.08.001.

[18] G. Björkman and A. Klarbring. Shakedown and residual stresses in frictional systems. In G M L Gladwell, H Ghonem and J Kalousek (Eds.), Contact Mechanics and Wear of Rail/Wheel Systems II: Proceedings of the 2nd International Symposium, pages 27-39. 1987.

[19] M.R. Brake, R.C. Flicek, and D.A. Hills. Development of a coupling metric to assess the shakedown limits for a contact interface. In 5th World Tribology Congress, Torino, Italy, September 2013. 
[20] A. Thaitirarot, R.C. Flicek, D.A. Hills, and J.R. Barber. The use of static reduction in the finite element solution of two-dimensional frictional contact problems. P. I. Mech. Eng. C-J. Mech., 228(9):1474-1487, June 2014. doi: $10.1177 / 0954406213509086$.

[21] J. Dundurs. Discussion of "Edge-bonded dissimilar or orthogonal elastic wedges under normal and shear loading". J. Appl. Mech., 36:650-652, 1969. doi: 10.1115/1.3564739.

[22] J.R. Barber. Elasticity, volume 172. Springer, 2009.

[23] K.L. Johnson. Contact Mechanics. Cambridge University Press, 1987.

[24] D.A. Hills, D. Nowell, and A. Sackfield. Mechanics of Elastic Contacts. Butterworth-Heinemann, Oxford, 1993.

[25] S. Topping and M. R. Brake. Developing and implementing an algorithm to characterize the signal robustness of strong links. 5321010-PR (OUO), Sandia National Laboratories, Albuquerque, NM, 2013.

[26] S. D. Topping, B. Simmons, M. R. Brake, and J. W. Wagner. Characerization of signals in electromechanical components. Unpublished Report (OUO), Sandia National Laboratories, Albuquerque, NM, 2014. 